Article

\title{
In Silico Screening of Potential Phytocompounds from Several Herbs against SARS-CoV-2 Indian Delta Variant B.1.617.2 to Inhibit the Spike Glycoprotein Trimer
}

\author{
Muruganantham Bharathi ${ }^{1}$ (D), Bhagavathi Sundaram Sivamaruthi ${ }^{1,2, *(D)}$, Periyanaina Kesika ${ }^{1,2}$ (D), \\ Subramanian Thangaleela ${ }^{1, *}$ and Chaiyavat Chaiyasut ${ }^{1, * \mathbb{D}}$ \\ 1 Innovation Center for Holistic Health, Nutraceuticals and Cosmeceuticals, Faculty of Pharmacy, \\ Chiang Mai University, Chiang Mai 50200, Thailand; bharathi.m03@gmail.com (M.B.); \\ p.kesika@gmail.com (P.K.) \\ 2 Office of Research Administration, Chiang Mai University, Chiang Mai 50200, Thailand \\ * Correspondence: sivamaruthi.b@cmu.ac.th (B.S.S.); leelasubramanian@gmail.com (S.T.); \\ chaiyavat@gmail.com (C.C.); Tel.: +66-53-944-340 (C.C.)
}

check for updates

Citation: Bharathi, M.; Sivamaruthi, B.S.; Kesika, P.; Thangaleela, S.; Chaiyasut, C. In Silico Screening of Potential Phytocompounds from Several Herbs against SARS-CoV-2 Indian Delta Variant B.1.617.2 to Inhibit the Spike Glycoprotein Trimer. Appl. Sci. 2022, 12, 665. https:// doi.org/10.3390/app12020665

Academic Editor: Panagiotis G Asteris

Received: 29 November 2021 Accepted: 1 January 2022

Published: 11 January 2022

Publisher's Note: MDPI stays neutral with regard to jurisdictional claims in published maps and institutional affiliations.

Copyright: (C) 2022 by the authors. Licensee MDPI, Basel, Switzerland. This article is an open access article distributed under the terms and conditions of the Creative Commons Attribution (CC BY) license (https:// creativecommons.org/licenses/by/ $4.0 /)$.

\begin{abstract}
In October 2020, the SARS-CoV-2 B.1.617 lineage was discovered in India. It has since become a prominent variant in several Indian regions and 156 countries, including the United States of America. The lineage B.1.617.2 is termed the delta variant, harboring diverse spike mutations in the N-terminal domain (NTD) and the receptor-binding domain (RBD), which may heighten its immune evasion potentiality and cause it to be more transmissible than other variants. As a result, it has sparked substantial scientific investigation into the development of effective vaccinations and anti-viral drugs. Several efforts have been made to examine ancient medicinal herbs known for their health benefits and immune-boosting action against SARS-CoV-2, including repurposing existing FDA-approved anti-viral drugs. No efficient anti-viral drugs are available against the SARS-CoV-2 Indian delta variant B.1.617.2. In this study, efforts were made to shed light on the potential of 603 phytocompounds from 22 plant species to inhibit the Indian delta variant B.1.617.2. We also compared these compounds with the standard drug ceftriaxone, which was already suggested as a beneficial drug in COVID-19 treatment; these compounds were compared with other FDAapproved drugs: remdesivir, chloroquine, hydroxy-chloroquine, lopinavir, and ritonavir. From the analysis, the identified phytocompounds acteoside $(-7.3 \mathrm{kcal} / \mathrm{mol})$ and verbascoside $(-7.1 \mathrm{kcal} / \mathrm{mol})$, from the plants Clerodendrum serratum and Houttuynia cordata, evidenced a strong inhibitory effect against the mutated NTD (MT-NTD). In addition, the phytocompounds kanzonol V $(-6.8 \mathrm{kcal} / \mathrm{mol})$, progeldanamycin $(-6.4 \mathrm{kcal} / \mathrm{mol})$, and rhodoxanthin $(-7.5 \mathrm{kcal} / \mathrm{mol})$, from the plant Houttuynia cordata, manifested significant prohibition against RBD. Nevertheless, the standard drug, ceftriaxone, signals less inhibitory effect against MT-NTD and RBD with binding affinities of $-6.3 \mathrm{kcal} / \mathrm{mol}$ and $-6.5 \mathrm{kcal} / \mathrm{mol}$, respectively. In this study, we also emphasized the pharmacological properties of the plants, which contain the screened phytocompounds. Our research could be used as a lead for future drug design to develop anti-viral drugs, as well as for preening the Siddha formulation to control the Indian delta variant B.1.617.2 and other future SARS-CoV-2 variants.
\end{abstract}

Keywords: delta variant B.1.617.2; Clerodendrum serratum; Houttuynia cordata; Siddha; mutated NTD; anti-viral drug

\section{Introduction}

Severe acute respiratory syndrome coronavirus 2 (SARS-CoV-2) first emerged in December 2019 and has since spread to over 221 nations, leading the continuing outbreak to be declared a worldwide medical emergency [1,2]. As of 17 December 2021, there have been more than 271.96 million confirmed cases and about 5.33 million deaths reported across almost 200 countries [3]. The treatment for individuals infected with SARS-CoV-2 
has garnered considerable attention. During COVID-19 outbreaks, significant numbers of possible SARS-CoV-2 treatments were postulated, such as inhibiting viral RNA synthesis, limiting virus replication, obstructing viral adherence to human cell surface receptors and reducing the virus's auto-assembly mechanism, all of which are the most effective anti-coronaviral methods [4-7]. The World Health Organization (WHO) instituted the SOLIDARITY study to investigate the four most viable COVID-19 treatment methods: remdesivir, chloroquine and hydroxy-chloroquine, lopinavir plus ritonavir, as well as lopinavir plus ritonavir and interferon- $\beta$ [3]. It is crucial to note that each of these four SARS-CoV-2 treatments targets one of the three coronavirus nonstructural proteins (NSPs): Mpro, RdRp, and PLpro [8-11]. SARS-CoV-2 has evolved, with multiple variations, which quickly established the dominant lineage B.1.617 [12,13]. B.1.617.1 was the first ascertained sublineage, accompanied by B.1.617.2, both of which have a similar L452R spike receptorbinding motif mutation [12-17].

The variation B.1.617.2 has been identified as a variant of concern (VOC) worldwide due to its apparent enhanced transmissibility, which has led to a rapid wave of infection devastating the Indian subcontinent $[18,19]$. This alteration was previously linked to enhanced disease transmission and a slight sensitivity to neutralizing antibodies. Since then, the delta variation B.1.617.2 has triumphed over the kappa variant B.1.617.1 and other lineages [20]. Following the discovery of the delta variant B.1.617.2, India reported a significant increase in COVID-19 cases, with the majority of cases and fatalities totaling over half a million by 6 May 2021, and over 6000 on 9 June 2021, which spread to at least 90 countries [21,22]. By 16 December 2020, countries such as the United States of America $(37.0 \%)$, the United Kingdom (11.0\%), Turkey $(8.0 \%)$, Germany $(5.0 \%)$, and Denmark $(5.0 \%)$ had detected the delta variant with travel history from India [23]. Many nations implemented travel restrictions to and from India in reaction to the exceptionally high number of COVID-19 cases in India in order to stop the spread of the new strains [24,25].

The Indian delta variant B.1.617.2 infectivity, human-to-human transmission, pathogenesis, and immune evasion have mostly been demonstrated to be affected by naturally evolved mutations in the receptor-binding domain (RBD) [26,27]. The RBD mutations L452R, T478K, and E484Q were especially found in lineage B.1.617.2 and were described as the strains' hallmark alterations [28,29]. The mutations L452R, T478K, E484Q, D614G, and P681R in the spike protein, including inside the receptor-binding domain, were also reported as a globally circulating lineage $[30,31]$. Although found within the RBD and insensitive to several monoclonal antibodies (mAbs), the L452R, T478K, and E484Q alterations are of special significance [32,33]. These mutations have shown an upsurge in viral transmissibility from $18.6 \%$ to $24 \%$. While compared to the Wuhan-originated or wild-type SARS-CoV-2, the neutralizing ability towards B.1.617.2 was shown to be 5.8 times lower in recent research $[34,35]$. Likewise, spike mutations in the N-terminal domain (NTD) of the lineage B.1.617.2 increase immune evasion capacity [36,37].

Secondly, a shred of rising evidence has demonstrated that the NTD is a viable target for therapeutic and vaccination strategies [38,39]. In addition, recent research revealed that numerous neutralizing antibodies adhered to the S1-NTD exclusively, preventing its communication with the host cells $[40,41]$. Li et al. reported that the alteration N234Q was unusually resistant to nullifying antibodies; conversely, N165Q grew increasingly susceptible. Based on this scientific evidence and information, new drugs can be developed by interacting with the S1-NTD [42]. The continued spread of the highly transmissible SARSCoV-2 delta strain underscores the necessity of getting vaccinated against COVID-19 [43,44]. However, current research reported that with two doses of the Pfizer/BioNTech vaccination the real efficacy slightly lowered from $93.4 \%$ to $87.9 \%$ against B.1.1.7 and B.1.617.2, according to a negative case-control study carried out in England [45]. The AstraZeneca vaccine efficacy was substantially reduced from $66.1 \%$ to $59.8 \%$ against B.1.617.2 [46]. The emergence of viral variations that may evade the immunological response given by vaccinations has created a different problem [47]. As a result, discovering a treatment for SARS-CoV-2 infections is still necessary. The presented study suggests that repurposing and exploiting 
naturally occurring chemicals employing in silico techniques might be promising treatments for COVID-19, achieved within a short timeframe and at a reasonable price [48-56].

Siddha and Ayurvedic medicine, which originated in Tamil Nadu, South India, are two of the oldest traditional remedies [57-59]. The government of India has recommended Kabasura kudineer choornam for combating a COVID-19 viral infection. Since the lungs are the primary organ of kapha, Kabasura kudineer choornam is a polyherbal Siddha composition that includes 15 herbs suggested to manage typical respiratory illnesses, such as colds, coughs, breathing difficulties, and the flu [60-62]. In silico investigations revealed that the Kabasura kudineer formulation had anti-inflammatory, antipyretic, and antibacterial properties; as well, it poses a better ability to bind to the SARS-CoV-2 spike protein $[63,64]$. Likewise, Houttuynia cordata (H. cordata) played a vital role in herbal therapy for the SARS outbreak in Southern China in 2003 [65]. Furthermore, it acts effectively against chikungunya, human noroviruses, human herpes viruses, the dengue virus, influenza, the pseudorabies virus, and murine coronaviruses [66,67]. Das et al. discovered $H$. Cordata phytocompounds to be a potential inhibitor for Mpro and PLpro, thereby preventing the replication of SARS-CoV-2 [68]. Similarly, the dried flower bud Syzygium aromaticum (S. aromaticum), a plant indicated by the English name "clove," acts effectively against COVID-19 [69]. Spices such as cloves are used in three forms: whole dried buds, powdered cloves, or extracted as an essential oil. According to earlier literature research, additional noteworthy qualities include the ability to treat colds, cough, asthma, and upper respiratory diseases, as well as anti-cancer, anti-inflammatory, and antimutagenic activity [70]. Tallei et al. reported that the phytocompounds hesperidin, nabiximols, pectolinarin, epigallocatechin gallate, and rhoifolin from Citrus spp. are effective against the Mpro and the spike glycoprotein trimmer (S-Protein) of SARS-CoV-2, which inhibits proliferation of the virus [71]. According to Khazdair et al., Nigella sativa (N. sativa) has protective effects on obstructive lung disorders, and this herb might be helpful in the treatment of COVID-19 [72].

The phytocompounds of N. sativa impede viral entrance and reproduction within the host cell by interfering with its binding to ACE2 receptors [73,74]. Interestingly, Shree et al. stated that three phytoconstituents from Ocimum basilicum (O. basilicum) vicenin, sorientin 4'-O-glucoside 2"-O-p-hydroxy-benzoagte, and ursolic acid inhibited the Mpro of SARSCoV2 [75]. Likewise, consuming Piper nigrum (black pepper) (P. nigrum) or piperine may help limit viral growth [76,77]. The Ministry of AYUSH, Government of India, also described that black pepper might have an anti-SARS-CoV-2 role [78]. Metastasio et al. concluded from their study that short-term kratom usage might reduce pain associated with COVID-19 infection without causing physical or psychological withdrawal symptoms when the kratom was halted [79]. Therefore, considering the therapeutic importance of Kabasura kudineer, H. cordata, S. aromaticum, Citrus spp., N. sativa, O. basilicum, P. nigrum, and Mitragyna speciosa Korthi (M. speciosa Korthi) and their strong ethnopharmacological background, the present study is primarily intended to perform molecular docking studies with these crucial phytocompounds acting against the S-protein of the SARS-CoV-2 Indian delta variant B.1.617.2.

We performed the molecular docking studies using Autodock Vina with Pyrx v0.8 platform [80], Pymol v2.5 [81], Ligplot+ v2.2.4 [82], and Discovery Studio Visualizer v21.1.0.20298 (www.accelerys.com) (accessed on 4 November 2021). We also performed a drug-likeness, adsorption, digestion, metabolism, excretion, toxicity (ADMET), toxicity class, and lethal dosage study of the shortlisted phytocompounds using the Molinspiration server, ADMETlab 2.0 [83], and ProTox-II to evaluate the pharmacokinetics and medicinal chemistry ease of the screened bioactive phytocompounds [84]. 


\section{Materials and Methods}

\subsection{Phytocompounds from Kabasura Kudineer Choornam and Herbal Plants}

The phytocompounds from the Siddha classical formulations Kabasura kudineer choornam (15 ingredients of herbs Zingiber officinale (Z. officinale), Piper longum (P. longum), S. aromaticum, Tragia involucrate Linn. (T. involucrate L.), Anacyclus pyrethrum (A. pyrethrum), Hygrophilla auriculata (H. auriculata), Terminalia chebula (T. chebula), Adathoda vasica (A. vasica), Coleus amboinicus (C. amboinicus), Saussurea lappa (S. lappa), Tinospora cordifolia (T. cordifolia), Clerodendrum serratum (C. serratum), Andrographis paniculate (A. paniculata), Sida acuta (S. acuta), Cyperus rotundus (C. rotundus) [85,86]), H. cordata, S. aromaticum, Citrus spp., N. sativa, O. basilicum, P. nigrum Linn, and M. speciosa Korthi were subjected to an evaluation of their interactions with the S-protein of the Indian delta variant B.1.617.2.

\subsection{Target Preparation and Ligand Library}

The cryo-electron microscopy structure of the S-Protein, a subunit vaccine candidate for COVID-19 PDB: 7E7B [87], was downloaded from the protein data bank and edited to remove unnecessarily bounded ligands and water molecules using the Discovery Studio Visualizer v19.1.0.18287 (www.accelerys.com) (accessed on 4 November 2021) and saved in PDB format. The major phytoconstituents present in Kabasura kudineer choornam and the other selected herbs were retrieved in SDF file format, and some compounds in 2D structures were also obtained from the PubChem database. A 3D structure was delineated for each of the obtained 2D structures and optimized with a force field based on Chemistry at Harvard Macromolecular Mechanics (CHARMM) parameterization using ACD/Chemsketch vC05E41 (Advanced Chemistry Development, Inc., Toronto, ON, Canada). The 3D structures were saved in the SDF file format, and all the obtained phytocompounds were then converted into PDB file format using OPEN BABEL software [88].

\subsection{Mutated NTD Model}

In this study, the NTD domain from PDB: 7E7B was mutated with Asn165Gln and Asn234Gln. No significant conformational change was observed in the structure of the mutant NTD model. Structural evaluation with RAMPAGE showed similar residue numbers in the most favored region (96.81\%). Structural alignment and superimposition of wild type (WT-NTD) and mutant type (MT-NTD) (Asn165Gln and Asn234Gln) were performed with the 3D-SS server (http:/ / cluster.physics.iisc.ernet.in/3dss/) (accessed on 26 October 2021) to calculate the disparity in the mutated sites. The superimposed structure was visualized in the Discovery Studio Visualizer v19.1.0.18287 software.

\subsection{Molecular Docking}

After preparing the phytocompounds as ligands and the receptor RBD and MT-NTD from 7E7B as targets, PyRx was implied with the Autodock Vina option using the new scoring function [89]. It analyzes the docking propensity and interfaces between the ligands, RBD, and mutated NTD. The prepared targets and ligands were converted into a PDBQT file format. For our docking analysis, we applied the specific search anchoring function of the PyRxVirtual Screening tool. The grid box properties were set as size_x $=31.43 \AA$, $y=46.11 \AA$, and size $\_z=32.15 \AA$ for the NTD molecular docking and size $\_x=36.38 \AA$, size_y $=67.04 \AA$, and size $\_z=32.08 \AA$ was set for the RBD molecular docking and then docked. The ligands were screened out for a binding affinity of $\leq 6.0 \mathrm{kcal} / \mathrm{mol}$. The significant interaction between the ligands and the receptors' binding site was acquired in 2D and 3D formats by importing the docked results into the LigPlot+, PyMol, and Discovery Studio Visualizer v19.1.0.18287 (www.accelerys.com) (accessed on 4 November 2021). In the autodock vina scoring function, 


$$
C=\sum_{i<j} f_{t i t j\left(r_{i j}\right)}
$$

where $C$ is sum of intermolecular and intramolecular distance; $\sum$ is the overall pairs of atoms; $f_{t i t j}$ is symmetric set of interaction functions; and $r_{i j}$ is interatomic distance.

\subsection{Evaluation of Ligands Drug Likeness and Toxicity}

The screened ligands were evaluated for draggability, physicochemical properties, toxicity, toxicity classes, and lethal dose using the Molinspiration server (www.molinspiration. $\mathrm{com} /$ cgi-bin/properties) (accessed on 4 November 2021). The druggability properties were analyzed based on the molar weights (MW), total polar surface area (TPSA), lipophilicity $(\log \mathrm{P})$, hydrogen bond acceptor (HBA), and hydrogen bond donor (HBD) to identify Lipinski's rule of the drug-like compounds. In addition, the simplified molecular-input line-entry system (SMILES) was downloaded from the PubChem Database to calculate the ADMET properties with toxicity class. The ADMET properties were calculated by implementing ADMETlab 2.0 [83] and ProTox-II with default parameters [84].

\section{Results}

The cryo-electron microscopy structure of the S-Protein trimer, a subunit vaccine candidate for COVID-19 PDB: 7E7B [87], is depicted in Figure 1a, containing chain A, chain B and chain C. Chain A was separated from PDB, and the 7E7B S-Protein trimmer contains the three major units: NTD (14-305), RBD (329-521), and S2 subunit (522-1147), as described in Figure 1b. The structure of the MT-NTD and the mutation in the RBD are pictured in Figure 1c.

The MT-NTD structure was compared with the WT-NTD, and the structural variation and coordination were evaluated. The WT-NTD (Figure 2a) and MT-NTD (Figure 2b) were validated using the Ramachandran plot. For the WT-NTD and MT-NTD, 98.92\% residues were present in the most favored region, and no significant changes were noted between the WT-NTD and MT-NTD. The RMSD and sequence identity were compared for the superimposed structure of the MT-NTD with WT-NTD. The RMSD and sequence identity of the MT-NTD was $0.004 \AA$, and $99.68 \%$, while the WT-NTD was set as a fixed molecule. Furthermore, the stamp score was noted as 9.799 for the MT-NTD out of 10 . The superimposed WT-NTD and MT-NTD structures' stamp sequence alignment is shown in Figure S1 (Supplementary File). The change in two amino acids did not produce any considerable alteration in the overall structural conformation of the protein in terms of the smaller RMSD and sequence identity. The superimposition of binding residues for the WT-NTD (Asn165, Asn234) and MT-NTD (Gln165, Gln234) models are shown in Figure 2c, respectively.

We used 603 phytocompounds in the screening process, obtained from the 22 wellannotated herbal plants included with the Kabasura kudineer choornam (Figure 3a). After processed molecular docking, the ligands with a higher binding affinity $(\leq 6.0 \mathrm{kcal} / \mathrm{mol})$ were screened from the docked results (Figure $3 b$ ).

The effects of phytocompounds from the plants H. cordata, Citrus spp., N. sativa, O. basilicum, P. nigrum, M. speciosa Korthi, and Kabasura kudineer, including 15 herbs, were analyzed to understand the binding efficacy against the targets $\mathrm{N}$-domain and RBD domain. The docked phytocompounds with the binding affinity $\leq-6.0 \mathrm{kcal} / \mathrm{mol}$ were predicted and listed separately for the MT-NTD and RBD. The phytocompounds from H. cordata, S. aromaticum, M. speciosa Korth, C. serratum, H. auriculata, Andrographis paniculata (A. paniculata), M. cerviana, T. involucrata, and T. cordifolia effectively inhibited the mutated N-Domain. 
A)

PDB: 7E7B

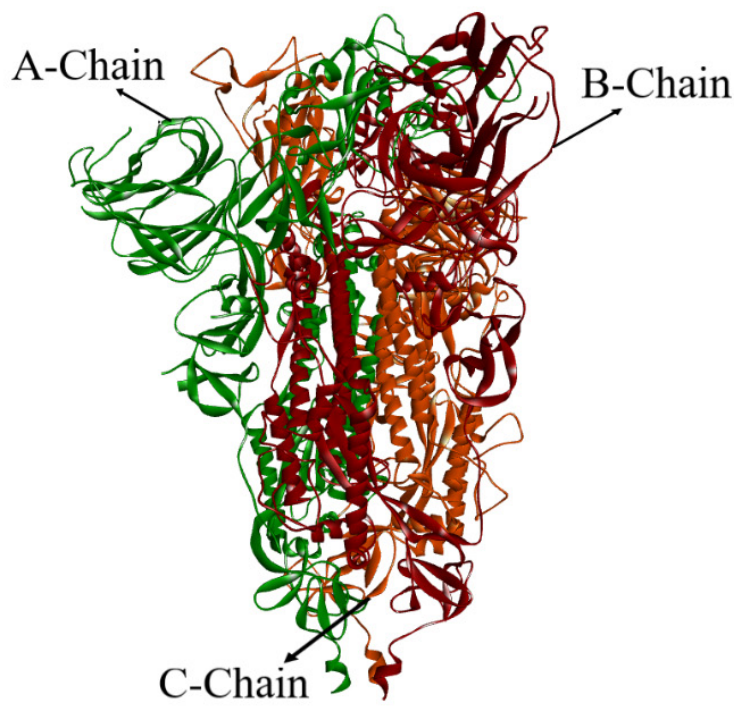

SARS-CoV-2 furin site mutant

$\mathrm{S}$-Trimer- a subunit vaccine candidate
B)

PDB: 7E7B : Chain A

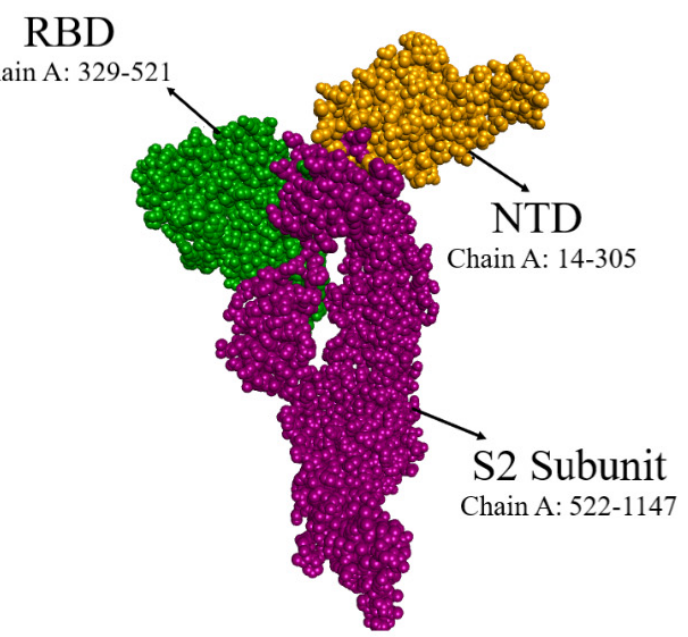

SARS-CoV-2 furin site mutant

S-Trimer- a subunit vaccine candidate: Chain A

C)

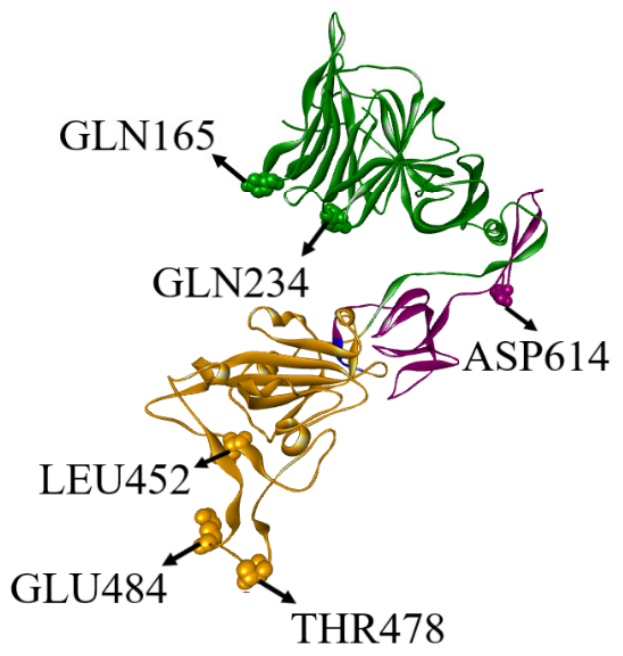

SARS-CoV-2 furin site mutant

S-Trimer- a subunit vaccine candidate

: Chain A with NTD mutation

Figure 1. The structure of the study protein PDB:7E7B. (A) The structure of SARS-CoV-2 S-protein (PDB: 7E7B) of B.1.67.2. A vaccine candidate constitutes Chain A, B, and C. (B) The structure of S-protein chain A contains NTD, RBD, and S2 subunit. (C) The structure of MT-NTD domain and RBD with its mutation as Indian delta variant B.1.617.2 S-Protein (Green-MT-NTD domain; purple-S2 subunit; yellow-RBD; the mutated residues of MT-NTD and RBD domains' crucial residues are highlighted as Corey-Pauling-Koltun (CPK) surface structure). 
A)

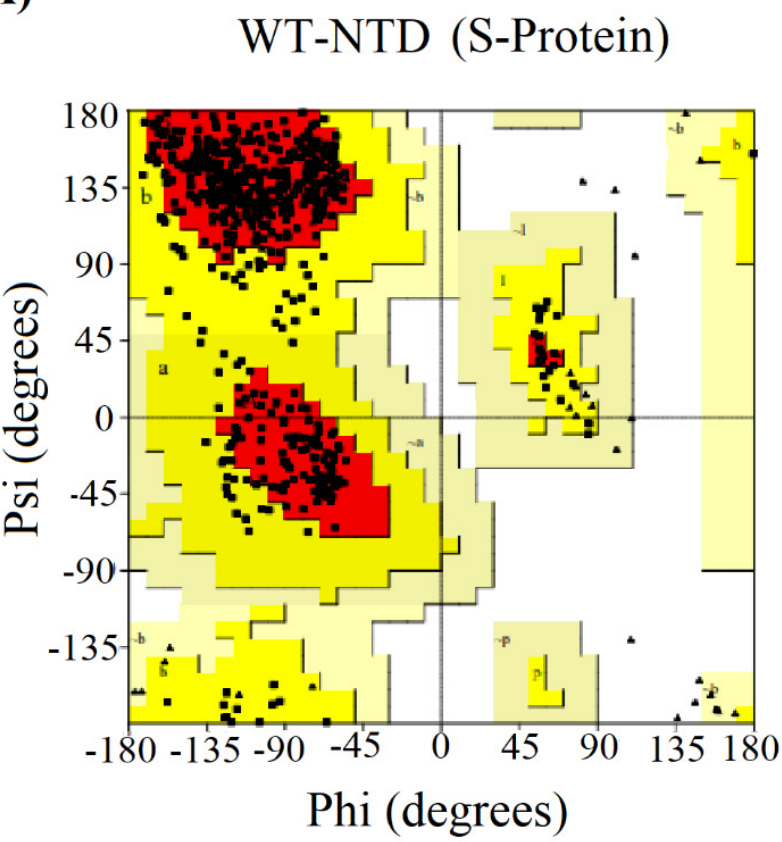

B)

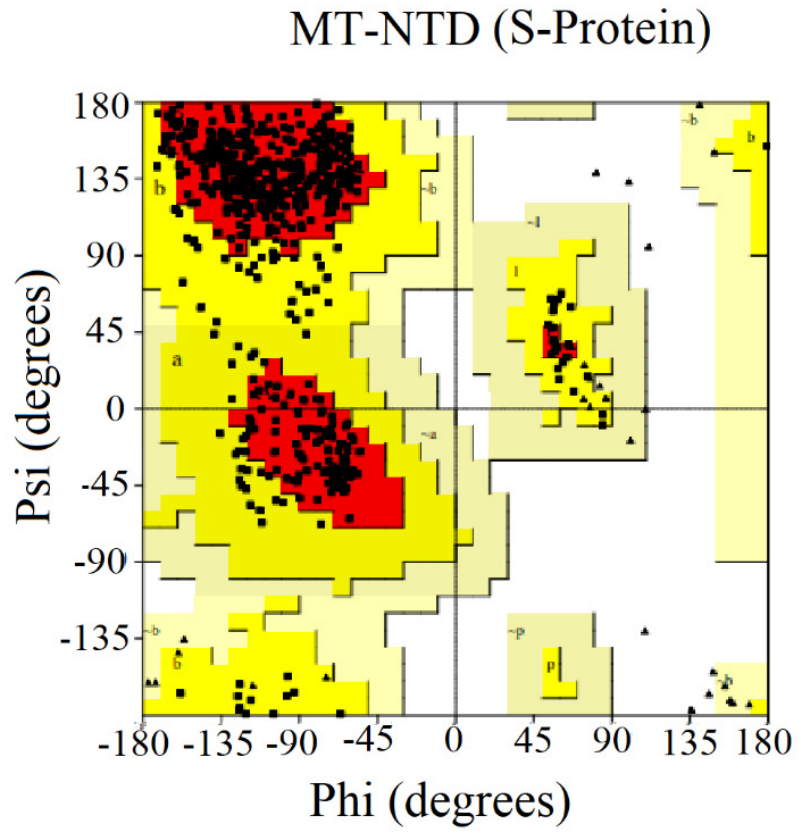

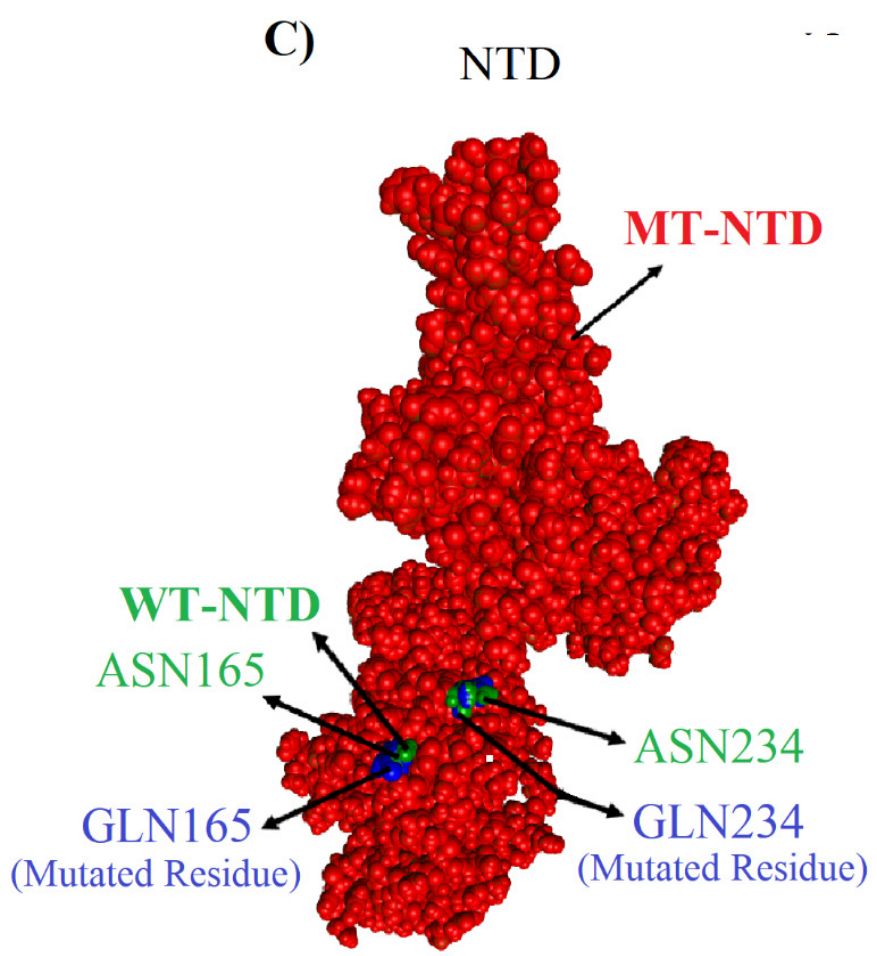

Figure 2. The B.1.617.2 S-protein wild- and mutated-type structure evaluation and comparison by implementing Ramachandran plot. (A) Ramachandran plot for the wild-type S-protein structure (B) NTD-mutated S-protein structure (C) The superimposed structure of WT-NTD and MT-NTD (Green-WT-NTD; red-MT-NTD; green and blue (CPK surface)—normal and mutated residues. 


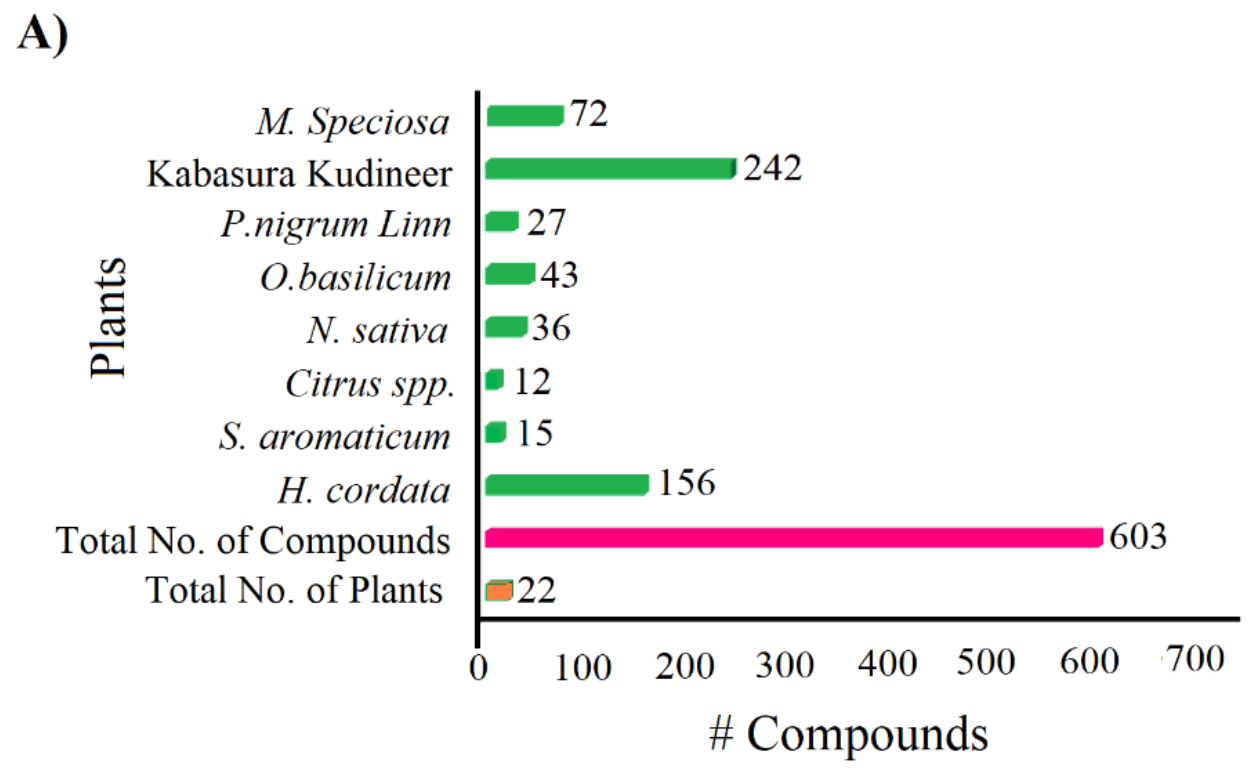

B)

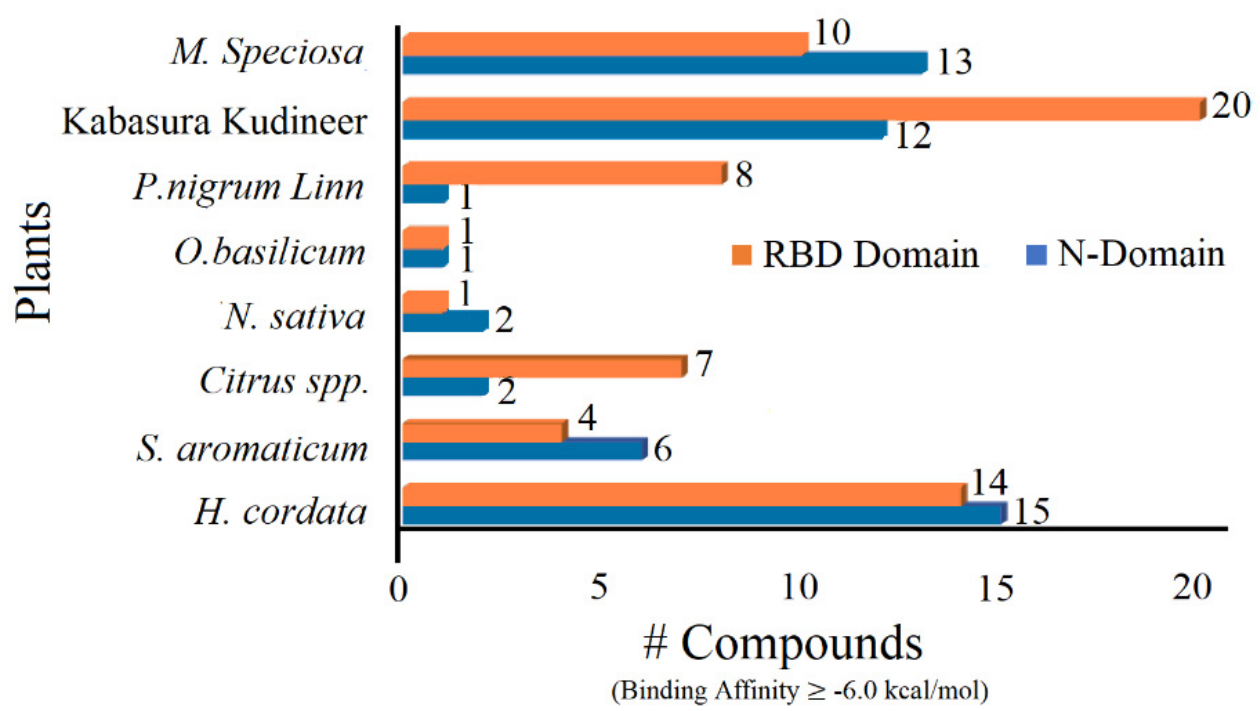

Figure 3. Depiction of the total number of plants, phytocompounds, and the phytocompounds with $\leq-6.0 \mathrm{kcal} / \mathrm{mol}$ binding energy. (A) The total number of plants and phytocompounds utilized in molecular docking study. (B) The number of phytocompounds with the $\leq-6.0 \mathrm{kcal} / \mathrm{mol}$ binding energy for the further screening process.

The chemical properties, including the molecular formula, molecular weight, and PubChem ID for the phytocompounds acted significantly against the NTD and RBD (binding affinity $\leq-6.0 \mathrm{kcal} / \mathrm{mol}$ ), listed in Tables S1-S4 (Supplementary File S1). The phytocompounds that beneficially interacted with the mutated NTD domain, its binding affinity, and LigPlot interactions are listed in Table 1 and Figure S2 (Supplementary File S1). 
Table 1. Binding affinity, RMSD, and interacting residues of the screened phytocompounds against mutated NTD domain.

\begin{tabular}{|c|c|c|c|c|c|c|c|c|}
\hline Plant & Phytocompounds & $\begin{array}{l}\text { Binding } \\
\text { Affinity }\end{array}$ & $\begin{array}{l}\text { RMSD } \\
\text { (̊) }\end{array}$ & $\begin{array}{l}\mathrm{H} / \mathrm{C}-\mathrm{H} \text { Bond } \\
\text { Interaction }\end{array}$ & $\begin{array}{l}\text { Bond } \\
\text { Length }\end{array}$ & $\begin{array}{l}\text { Hydrophobic } \\
\text { Interaction }\end{array}$ & $\begin{array}{l}\text { Alkyl } \\
\text { Interaction }\end{array}$ & $\begin{array}{l}\text { Pi-Sigma/ } \\
\text { Cation } \\
\text { Stacked } \\
\text { Interaction }\end{array}$ \\
\hline \multicolumn{9}{|c|}{ B.1.617.2. S-Protein-N-Domain (Mutant Type) } \\
\hline $\begin{array}{l}\text { Standard } \\
\text { Drug }\end{array}$ & Ceftriaxone & -6.3 & 1.625 & $\begin{array}{l}\text { ASP88, ASN87, } \\
\text { GLN115, } \\
\text { ASN156* ASP198, } \\
\text { GLY199, GLY232, } \\
\text { GLN234*, }\end{array}$ & $\begin{array}{l}2.57,2.23 \\
2.10,2.54 \\
2.03,2.76, \\
2.28 .2 .93\end{array}$ & ASN196, ILE197 & ILE233 & - \\
\hline \multirow{9}{*}{ H. cordata } & $\begin{array}{l}\text { Cholest-4,14-dien- } \\
\text { 15,20-diol-3,16- } \\
\text { dione }\end{array}$ & -6 & 2.414 & $\begin{array}{l}\text { ASN196, } \\
\text { GLN234 }\end{array}$ & $5.03,4.72$ & $\begin{array}{l}\text { ASP88, ASP198, } \\
\text { GLY199, GLY232, } \\
\text { ILE233, ILE235 }\end{array}$ & $\begin{array}{l}\text { LEU54, } \\
\text { ILE197 }\end{array}$ & - \\
\hline & Dihydrocelastrol & -6.6 & 1.41 & $\begin{array}{l}\text { ASP53, ASN196, } \\
\text { GLN234, ILE235 }\end{array}$ & $\begin{array}{l}4.56,3.63 \\
4.74,4.38\end{array}$ & $\begin{array}{l}\text { ASN87, ASP88, } \\
\text { LYS195, ILE233 }\end{array}$ & $\begin{array}{l}\text { LEU54, } \\
\text { ILE197 }\end{array}$ & \\
\hline & Isoquercitrin & -6.4 & 2.445 & $\begin{array}{l}\text { ASN196, ILE233, } \\
\text { GLN234, ILE235, } \\
\text { ASN87* }\end{array}$ & $\begin{array}{l}4.40,5.36 \\
4.45,5.41\end{array}$ & $\begin{array}{l}\text { LEU54, PHE86, } \\
\text { ILE197, ASP198, } \\
\text { GLY199, THR236 }\end{array}$ & & ASP88 \\
\hline & Naltrindole & -6.3 & 2.726 & & & $\begin{array}{l}\text { ASN87, ILE197, } \\
\text { ASP198, ILE233, } \\
\text { ILE235, THR236 }\end{array}$ & $\begin{array}{l}\text { GLN234, } \\
\text { ASN1196, } \\
\text { LEU54, } \\
\text { PRO272 }\end{array}$ & ASP88 \\
\hline & Pirenperone & -8.7 & 1.395 & ILE233 & - & $\begin{array}{l}\text { ASN87, ASP88, } \\
\text { ASN196, GLT199, } \\
\text { GLY232, GLN234, } \\
\text { PRO272 }\end{array}$ & ILE197 & LEU54 \\
\hline & Quercitrin & -6.3 & 1.588 & $\begin{array}{l}\text { ASN87, ASP88, } \\
\text { ASN196, } \\
\text { GLN234, ILE235 }\end{array}$ & $\begin{array}{l}4.54,3.67 \\
4.74,4.47 \\
5.69,3.46\end{array}$ & $\begin{array}{l}\text { LEU54, PHE86, } \\
\text { ASP198, GLY199, } \\
\text { ILE233 }\end{array}$ & - & - \\
\hline & Rhodoxanthin & -6.8 & 2.258 & ASP198 & 然 & $\begin{array}{l}\text { THR114, GLN115, } \\
\text { GLU132, GLN165, } \\
\text { CYS166, THR167, } \\
\text { ASN196, ILE197, } \\
\text { GLY199, GLY232, } \\
\text { ILE233, GLN234 }\end{array}$ & - & - \\
\hline & Sesamin & -9.1 & 0.046 & $\begin{array}{l}\text { ASN196*, } \\
\text { ILE197*, ASP198 }\end{array}$ & - & $\begin{array}{l}\text { ASP88, ILE233, } \\
\text { GLN234, ILE235, } \\
\text { PRO272 }\end{array}$ & & LEU54 \\
\hline & Usambarensine & -6.8 & 1.9 & $\begin{array}{l}\text { ASN196, } \\
\text { ASP198*, ILE233* }\end{array}$ & 4.57 & $\begin{array}{l}\text { ASP88, GLY199, } \\
\text { GLN234, ILE235 }\end{array}$ & LEU54 & $\begin{array}{l}\text { ASP197, } \\
\text { ASP198 }\end{array}$ \\
\hline \multirow{4}{*}{ S. aromaticum } & Biflorin & -6 & 1.436 & $\begin{array}{l}\text { ASP88, ASN196, } \\
\text { GLN234, ILE235 } \\
\text { GLN234* }\end{array}$ & & $\begin{array}{l}\text { PHE86, ILE197, } \\
\text { ASP198, GLY199, } \\
\text { TYR200, ILE233, } \\
\text { THR236 }\end{array}$ & GLN234 & - \\
\hline & Crategolic acid & -6.4 & 1.764 & $\begin{array}{l}\text { ASN196, } \\
\text { GLN234 }\end{array}$ & $3.96,4.57$ & $\begin{array}{l}\text { LEU54, ASP88, } \\
\text { ILE197, ASP198, } \\
\text { GLY199, ILE235, } \\
\text { PRO272 }\end{array}$ & & \\
\hline & Oleanolic acid & -6.2 & 2.01 & ASN87*, ILE235 & & $\begin{array}{l}\text { ASP53, LEU54, } \\
\text { PHE86, ASP88, } \\
\text { ASN196, ILE197, } \\
\text { GLN234, THR236 }\end{array}$ & - & - \\
\hline & Rhamnetin & -6 & 1.785 & $\begin{array}{l}\text { ASN87, ASP88, } \\
\text { GLY199 }\end{array}$ & $\begin{array}{l}3.29,6.01 \\
3.40\end{array}$ & $\begin{array}{l}\text { PHE86, ASN196, } \\
\text { ASP198, GLY232, } \\
\text { ILE233, GLN234, } \\
\text { ILE235 }\end{array}$ & & \\
\hline
\end{tabular}


Table 1. Cont.

\begin{tabular}{|c|c|c|c|c|c|c|c|c|}
\hline Plant & Phytocompounds & $\begin{array}{l}\text { Binding } \\
\text { Affinity }\end{array}$ & $\begin{array}{l}\text { RMSD } \\
\text { (̊̊) }\end{array}$ & $\begin{array}{l}\text { H/C-H Bond } \\
\text { Interaction }\end{array}$ & $\begin{array}{l}\text { Bond } \\
\text { Length }\end{array}$ & $\begin{array}{l}\text { Hydrophobic } \\
\text { Interaction }\end{array}$ & $\begin{array}{l}\text { Alkyl } \\
\text { Interaction }\end{array}$ & $\begin{array}{l}\text { Pi-Sigma/ } \\
\text { Cation } \\
\text { Stacked } \\
\text { Interaction }\end{array}$ \\
\hline \multirow{3}{*}{$\begin{array}{l}\text { M. speciosa } \\
\text { Korthi }\end{array}$} & Chlorogenic acid & -6.1 & 2.307 & $\begin{array}{l}\text { PHE86, ASN196, } \\
\text { ILE235 }\end{array}$ & $\begin{array}{l}4.34,2.65, \\
5.55\end{array}$ & \multicolumn{3}{|l|}{$\begin{array}{l}\text { LEU54, ASP88, } \\
\text { ASN87, ILE197, } \\
\text { ASP198, GLY199, } \\
\text { TYR200, GLY232, } \\
\text { ILE233, GLN234, } \\
\text { THR236 }\end{array}$} \\
\hline & Isoquercitrin & -6.4 & 2.484 & $\begin{array}{l}\text { ASN196, ILE233, } \\
\text { GLN234, ILE235, } \\
\text { ASN87* }\end{array}$ & $\begin{array}{l}6.26^{*}, 4.25 \\
4.43,5.59 \\
4.47\end{array}$ & $\begin{array}{l}\text { LEU54, PHE86, } \\
\text { ILE197, ASP198, } \\
\text { GLY199, THR236 }\end{array}$ & - & ASP88 \\
\hline & Rutin & -6.1 & 2.149 & $\begin{array}{l}\text { ASN196, } \\
\text { ASN196*, } \\
\text { ASP198, } \\
\text { GLN234*, ILE235 }\end{array}$ & $\begin{array}{l}3.79,4.05^{*}, \\
3.85,3.67^{*} \\
5.01\end{array}$ & $\begin{array}{l}\text { PHE86, ASN87, } \\
\text { ASP88, GLY199, } \\
\text { GLY232, ILE233, } \\
\text { THR236 }\end{array}$ & $\begin{array}{l}\text { LEU54, } \\
\text { ILE197 }\end{array}$ & - \\
\hline \multicolumn{9}{|c|}{ Kabasura kudineer } \\
\hline \multirow{3}{*}{ C. serratum } & Acteoside & -7.3 & 2.804 & \multirow{3}{*}{$\begin{array}{l}\text { PHE86, ASN87, } \\
\text { ASP88, ASN196, } \\
\text { ASP198, GLY199, } \\
\text { ILE233, GLN234 } \\
\text { ASP88, GLN234 } \\
\text { ASN87, ASP88*, } \\
\text { ASN196, ASP198, } \\
\text { GLY199, ILE233, } \\
\text { GLN234 }\end{array}$} & $\begin{array}{l}4.45,6.04 \\
3.09,4.34 \\
3.47,5.84 \\
3.78\end{array}$ & $\begin{array}{l}\text { LEU54, LYS195, } \\
\text { THR236 }\end{array}$ & ILE197 & \\
\hline & Serratagenic acid & -6.8 & 2.404 & & $4.33,4.54$ & - & - & - \\
\hline & Verbascoside & -7.1 & 1.904 & & $\begin{array}{l}6.07,3.99 \\
3.49,4.04 \\
5.37,4.73\end{array}$ & $\begin{array}{l}\text { GLN52, PRO85, } \\
\text { ILE235 }\end{array}$ & $\begin{array}{l}\text { LEU54, } \\
\text { PRO272 }\end{array}$ & THR236 \\
\hline \multirow[b]{2}{*}{ H. auriculata } & $\begin{array}{l}\text { Apigenin } \\
\text { 7-O-glucoside }\end{array}$ & -6.5 & 2.588 & $\begin{array}{l}\text { ASN87, ASN196, } \\
\text { GLY199, } \\
\text { GLN234, ILE235 }\end{array}$ & $\begin{array}{l}5.13,3.54 \\
3.65,3.42, \\
3.95,4.52\end{array}$ & \multicolumn{2}{|l|}{$\begin{array}{l}\text { PHE86, ASP88, } \\
\text { ILE233 }\end{array}$} & ASP198 \\
\hline & Cucurbitacin B & -6.2 & 2.838 & GLN234, GLY199 & $3.34,4.84$ & $\begin{array}{l}\text { PHE86, ASN87, } \\
\text { ASP88, THR108, } \\
\text { THR114, ILE197, } \\
\text { ASP198, ILE233, } \\
\text { ILE235, THR236 }\end{array}$ & - & - \\
\hline A. paniculata & Neoandrographolide & -6.3 & 1.466 & $\begin{array}{l}\text { ASN87, ASN196, } \\
\text { GLN234, ILE235, } \\
\text { PRO272* }\end{array}$ & $\begin{array}{l}3.79,4.2 \\
5.13,4.25 \\
5.55\end{array}$ & $\begin{array}{l}\text { GLN52, ASP53, } \\
\text { LEU54, PHE86, } \\
\text { THR236 }\end{array}$ & & ASP88 \\
\hline \multirow[b]{2}{*}{ M. cerviana } & Orientin & -6.2 & 1.189 & $\begin{array}{l}\text { ASN87*, ASP88, } \\
\text { ASN196, ILE233, } \\
\text { GLN234, ILE235 }\end{array}$ & $\begin{array}{l}4.32,421 \\
5.42,4.98 \\
3.95\end{array}$ & $\begin{array}{l}\text { PHE86, ASN87, } \\
\text { ASP198, GLY199, } \\
\text { GLY232, THR236 }\end{array}$ & ILE197 & - \\
\hline & Vitexin & -6.2 & 1.562 & $\begin{array}{l}\text { ASN87, ASP88, } \\
\text { ASN196, } \\
\text { GLN234, } \\
\text { GLN234*, } \\
\text { ILE235, ASP198 }\end{array}$ & $\begin{array}{l}5.44,3.01 \\
3.24,3.52 \\
4.21\end{array}$ & 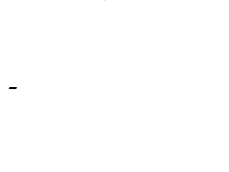 & - & - \\
\hline T. involucrate L. & Rutin & -6.4 & 1.702 & $\begin{array}{l}\text { ASP88*, ASN196, } \\
\text { ASP198, GLY199, } \\
\text { ILE233, GLN234 }\end{array}$ & $\begin{array}{l}4.39,2.93 \\
4.48,4.79\end{array}$ & \multirow{2}{*}{$\begin{array}{l}\text { PHE86, ASN87, } \\
\text { GLY89, ILE231, } \\
\text { GLY232, ILE235, } \\
\text { PRO272 } \\
\text { PHE86, ASP88, } \\
\text { ASN196, TYR200, } \\
\text { GLN234 }\end{array}$} & \multirow[t]{2}{*}{$\begin{array}{l}\text { LEU54, } \\
\text { ILE197 }\end{array}$} & \\
\hline T. cordifolia & Tinosporide & -6.1 & 2.551 & $\begin{array}{l}\text { ASN87*, ASP198, } \\
\text { GLY199, ILE233. } \\
\text { ILE235* }\end{array}$ & $\begin{array}{l}4.50,3.59 \\
5.90\end{array}$ & & & THR236 \\
\hline
\end{tabular}

Note: * indicates the carbon-hydrogen bond.

The screened phytocompounds binding affinity and its LigPlot interactions for the RBD domain are listed in Table 2 and Figure S3 (Supplementary File S1). The pharmacological activity of the resulting active compounds acts against the NTD and RBD is listed in Table 3, and the predicted drug-likeness and toxicity classes are presented in Tables 4 and 5 . 
Table 2. Binding affinity, RMSD, and interacting residues of the screened phytocompounds against RBD domain.

\begin{tabular}{|c|c|c|c|c|c|c|c|c|}
\hline Plant & Phytocompounds & $\begin{array}{l}\text { Binding } \\
\text { Affinity }\end{array}$ & $\begin{array}{l}\text { RMSD } \\
\text { (Å) }\end{array}$ & $\begin{array}{l}\mathrm{H} / \mathrm{C}-\mathrm{H} \text { Bond } \\
\text { Interaction }\end{array}$ & $\begin{array}{l}\text { Bond } \\
\text { Length }\end{array}$ & $\begin{array}{l}\text { Hydrophobic } \\
\text { Interaction }\end{array}$ & $\begin{array}{l}\text { Alkyl } \\
\text { Interaction }\end{array}$ & $\begin{array}{l}\text { Pi-Sigma/ } \\
\text { Cation } \\
\text { Stacked } \\
\text { Interaction }\end{array}$ \\
\hline \multicolumn{9}{|c|}{ B.1.617.2. S-Protein-RBD-Domain } \\
\hline $\begin{array}{l}\text { Standard } \\
\text { Drug }\end{array}$ & Ceftriaxone & -6.5 & 1.625 & $\begin{array}{l}\text { ARG457, LYS458, } \\
\text { GLU471*, } \\
\text { GLN474, CYS480 }\end{array}$ & $\begin{array}{l}3.45,5.86 \\
3.40,4.14 \\
3.52,3.59 \\
3.60\end{array}$ & $\begin{array}{l}\text { ARG454, GLU465, } \\
\text { ASP567, SER469, } \\
\text { TYR473, PRO479, } \\
\text { GLY482, PRO491 }\end{array}$ & CYS480 & ARG457 \\
\hline \multirow{6}{*}{ H. cordata } & $\begin{array}{l}\text { Cholest-4,14-dien- } \\
\text { 15,20-diol-3,16- } \\
\text { dione }\end{array}$ & -7.2 & 1.543 & SER494 & 4.08 & $\begin{array}{l}\text { ARG403, TYR449, } \\
\text { GLU484, GLN493, } \\
\text { SER494, TYR495, } \\
\text { GLY496, THR500, } \\
\text { ASN501, GLY502, } \\
\text { TYR505 } \\
\text { LEU452, GLU484, } \\
\text { GLY485, CYS488, } \\
\text { TYR489, LEU492, } \\
\text { GLN493 }\end{array}$ & - & PHE490 \\
\hline & $\begin{array}{l}\text { Fluorometholone } \\
17 \text {-acetate }\end{array}$ & -6.1 & 3.672 & PHE490, GLN493 & $4.54,5.00$ & $\begin{array}{l}\text { LEU452, PHE456, } \\
\text { GLU484, TYR489, } \\
\text { SER494 }\end{array}$ & - & LEU492 \\
\hline & Kanzonol V & -6.8 & 2.182 & GLU484, TYR449 & $4.54,4.55$ & $\begin{array}{l}\text { LEU452, LEU455, } \\
\text { PHE490, LEU492, } \\
\text { GLN493, SER494 }\end{array}$ & - & $\begin{array}{l}\text { PHE456, } \\
\text { TYR489 }\end{array}$ \\
\hline & Progeldanamycin & -6.4 & 2.392 & TYR449, SER494 & $3.45,2.62$ & $\begin{array}{l}\text { ASN450, PHE490, } \\
\text { LEU492, GLN493 }\end{array}$ & - & LEU452 \\
\hline & Rhodoxanthin & -7.5 & 1.856 & - & - & $\begin{array}{l}\text { ARG346, SER349, } \\
\text { TYR351, ASN450, } \\
\text { LEU455, PHE456, } \\
\text { GLU484, GLY485, } \\
\text { PHE486, LEU492, } \\
\text { GLN493, SER494 }\end{array}$ & $\begin{array}{l}\text { LEU452, } \\
\text { TYR489, } \\
\text { PHE490 }\end{array}$ & - \\
\hline & $\begin{array}{l}\text { Stigmastane-3,6- } \\
\text { dione, } \\
\text { (5.alpha) }\end{array}$ & -6.8 & 3.213 & GLN493, SER494 & $4.98,4.04$ & $\begin{array}{l}\text { TYR351, LEU452, } \\
\text { THR470, GLU484, } \\
\text { LEU492 }\end{array}$ & PHE490 & - \\
\hline S. aromaticum & Rhamnetin & -6.1 & 2.981 & $\begin{array}{l}\text { ARG346, SER349, } \\
\text { TRP353, SER349 }\end{array}$ & $\begin{array}{l}4.12,3.78 \\
4.73\end{array}$ & $\begin{array}{l}\text { PHE347, ARG355, } \\
\text { LEU452, ARG466 }\end{array}$ & $\begin{array}{l}\text { TYR351, } \\
\text { ALA352 }\end{array}$ & ALA348 \\
\hline \multirow{3}{*}{$\begin{array}{l}\text { M. speciosa } \\
\text { Korthi }\end{array}$} & Beta-Sitosterol & -6 & 1.586 & GLY485 & 3.45 & $\begin{array}{l}\text { GLU484, PHE486, } \\
\text { ASN487, LEU492, } \\
\text { GLN493, SER494 }\end{array}$ & $\begin{array}{l}\text { LEU452, } \\
\text { TYR489, } \\
\text { PHE490 }\end{array}$ & - \\
\hline & Stigmasterol & -6 & 3.456 & - & - & $\begin{array}{l}\text { LEU455, GLN484, } \\
\text { LEU492, GLN493 }\end{array}$ & $\begin{array}{l}\text { LEU452, } \\
\text { PHE456, } \\
\text { TYR489, }\end{array}$ & PHE490 \\
\hline & & & & & & & PHE490 & \\
\hline \multicolumn{9}{|c|}{ Kabasura kudineer } \\
\hline T. cordifolia & Berberine & -6.1 & 2.725 & $\begin{array}{l}\text { PHE490, } \\
\text { GLN493, SER494 }\end{array}$ & $\begin{array}{l}4.73,4.32, \\
4.15\end{array}$ & $\begin{array}{l}\text { TYR449, TYR489, } \\
\text { LEU492 } \\
\text { TYR449, LEU452, }\end{array}$ & LEU452 & - \\
\hline C. serratum & Clerodermic acid & -5.8 & 4.924 & GLU484 & $3.64,4.37$ & $\begin{array}{l}\text { THR470, LEU492, } \\
\text { GLN493, SER494 }\end{array}$ & - & PHE490 \\
\hline C. speciosus & Diosgenin & -6.9 & 1.058 & SER349 & 4.28 & $\begin{array}{l}\text { ARG346, PHE347, } \\
\text { ALA348, ASN450, } \\
\text { TRP353, ASN354, } \\
\text { ARG355, ARG466, } \\
\text { ILE468 }\end{array}$ & $\begin{array}{l}\text { ALA352, } \\
\text { LEU452 }\end{array}$ & - \\
\hline
\end{tabular}


Table 3. The pharmacological activity of the active compounds against S-Protein (mutated NTD and $\mathrm{RBD})$ of Indian delta variant B.1.617.2.

\begin{tabular}{|c|c|c|c|c|}
\hline Plant Name & Active Compounds & Plants Parts & Pharmacological Properties & References \\
\hline H. cordata & $\begin{array}{l}\text { Canthaxanthin, } \\
\text { Cholest-4,14-dien-15,20- } \\
\text { diol-3,16-dione, } \\
\text { Dihydrocelastrol, } \\
\text { Fluorometholone } \\
\text { 17-acetate, Isoquercitrin, } \\
\text { Kanzonol V, Naltrindole, } \\
\text { Pirenperone, } \\
\text { Progeldanamycin, } \\
\text { Quercitrin, Rhodoxanthin, } \\
\text { Sesamin, } \\
\text { Stigmastane-3,6-dione, } \\
\text { (5.alpha), Usambarensine }\end{array}$ & Whole Plant & $\begin{array}{l}\text { Cough, pneumonia, bronchitis, dysentery, dropsy, } \\
\text { leukorrhea, uteritis, eczema, herpes simplex, acne, } \\
\text { chronic sinusitis, stomach ulcer, infection, control } \\
\text { wrinkle, chapped skin, septic, febrifuge, heatstroke, } \\
\text { malaria, lung disorder, tonsillitis, skin ulcer, } \\
\text { diarrhea, dysentery arthritis, appendicitis, snake } \\
\text { bite, stomach disorder, sinusitis, heart disorders, } \\
\text { severe acute respiratory Syndrome (SARS), } \\
\text { chikungunya, herpes simplex viruses, dengue virus } \\
\text { serotype } 2 \text { (DEN-2), infuenza neuraminidase, } \\
\text { pseudorabies herpes virus (prv), human } \\
\text { noroviruses (hunovs), murine coronavirus and } \\
\text { dengue virus infection, innate immune modulation } \\
\text { activities, and inhibits the replication of SARS-CoV. }\end{array}$ & {$[65,90-98]$} \\
\hline S. aromaticum & $\begin{array}{l}\text { Biflorin, Crategolic acid, } \\
\text { Oleanolic acid, Rhamnetin }\end{array}$ & Cloves buds (Oil) & $\begin{array}{l}\text { Coughs, colds, asthma, respiratory and digestive } \\
\text { disorders, sinusitis, modulatory effects of cell } \\
\text { membrane permeability, acts against food borne } \\
\text { gram-positive bacteria, promotion of Go/G1 cell } \\
\text { cycle arrest, induction of apoptosis, anti-diabetic } \\
\text { activity, antioxidant, antitumor, cardio protective, } \\
\text { antifungal, and acts effectively against SARS-CoV-2. }\end{array}$ & {$[70,99-109]$} \\
\hline M. speciosa Korthi & $\begin{array}{l}\text { Chlorogenic acid, } \\
\text { Isoquercitrin, Rutin, } \\
\text { Beta-Sitosterol, } \\
\text { Stigmasterol }\end{array}$ & Leaves & $\begin{array}{l}\text { Tiredness and muscle fatigue, diarrhea, coughing, } \\
\text { muscle pain, anti-diabetic, wound, hypertension, } \\
\text { drug addiction, anti-inflammation, antinociceptive, } \\
\text { anti-oxidant, antimicrobial activity, and reduction } \\
\text { of muscle pain against SARS-CoV-2. }\end{array}$ & {$[79,110-115]$} \\
\hline \multicolumn{5}{|l|}{ Kabasura kudineer } \\
\hline C. serratum & $\begin{array}{l}\text { Acteoside, Clerodermic } \\
\text { acid, Serratagenic acid, } \\
\text { Verbascoside }\end{array}$ & Root & $\begin{array}{l}\text { Respiratory disease, fever, anti-inflammatory, } \\
\text { anticancer, antinociceptive, liver disorders, } \\
\text { anti-allergic, and acts as anti-oxidant. }\end{array}$ & {$[63,64,116,117]$} \\
\hline H. auriculata & Apigenin 7-O-glucoside & Root & $\begin{array}{l}\text { Anasarca, urinogenital tract disorder, hyperdipsia, } \\
\text { vesical calculi, flatulence, diarrhea, leukorrhea, } \\
\text { gonorrhea, gastrointestinal disorder, anti-tumor, } \\
\text { arthritis, painful micturition, menorrhagia, and } \\
\text { treats blood infection. }\end{array}$ & {$[63,64,118,119]$} \\
\hline T. cordifolia & Berberine, Tinosporide & Stem & $\begin{array}{l}\text { Immuno-modulation, pneumonia, asthma, cough, } \\
\text { swelling lungs, colic, constipation, tetanus, anthrax, } \\
\text { pox, fracture, antispasmodic, and } \\
\text { antipyretic activity. }\end{array}$ & {$[63,64,75,120,121]$} \\
\hline C. speciosus & Diosgenin & Root & $\begin{array}{l}\text { Pneumonia, constipation, skin diseases, fever, } \\
\text { asthma, bronchitis, inflammation, anaemia, dropsy, } \\
\text { cough, urinary diseases, jaundice, improves insulin } \\
\text { secretion, hypolipidemic, adaptogenic, anticancer, } \\
\text { and hepatoprotective activity. }\end{array}$ & {$[63,64,122-125]$} \\
\hline A. paniculata & Neoandrographolide & Whole plant & $\begin{array}{l}\text { Colds, sinusitis, influenza, immunostimulant, } \\
\text { anti-viral against hepatitis B, HIV, and respiratory } \\
\text { syncytial virus. }\end{array}$ & {$[63,64,126,127]$} \\
\hline M. cerviana & Orientin, Vitexin & Whole plant & $\begin{array}{l}\text { Anti-inflammatory, anti-oxidant, antimicrobial, } \\
\text { antidiabetic, hepatoprotective, photo-protective, } \\
\text { uterine stimulant, antiseptic, antipyretic, and } \\
\text { immunostimulant activity. }\end{array}$ & {$[63,64,128,129]$} \\
\hline T. involucrata & Rutin & Root & $\begin{array}{l}\text { High fever, inflammation, wounds, eczema, scabies, } \\
\text { skin infections, bronchitis pain, and } \\
\text { antimicrobial activity. }\end{array}$ & {$[63,64,121,130]$} \\
\hline
\end{tabular}


Table 4. Identification of drug-likeness and toxicity analysis for selected compounds inhibits mutated N-domain in S-Protein.

\begin{tabular}{|c|c|c|c|c|c|c|c|c|c|c|c|c|c|c|c|c|}
\hline \multicolumn{2}{|c|}{ Phytocompounds } & \multicolumn{5}{|c|}{ Drug-Likeness } & \multirow[b]{2}{*}{$\begin{array}{l}\text { No. of } \\
\text { Violations }\end{array}$} & \multicolumn{9}{|c|}{ Toxicity Analysis } \\
\hline Plant Name & Compound Name & $\operatorname{miLog} P$ & TPSA & Natoms & nON & nOHNH & & $\begin{array}{l}\text { Intestinal } \\
\text { Absorption }\end{array}$ & $\begin{array}{l}\text { Oral Toxicity } \\
\text { (- } \log \mathrm{kg} / \mathrm{mol})\end{array}$ & $\begin{array}{l}\text { Hepato } \\
\text { Toxicity }\end{array}$ & $\begin{array}{l}\text { Carcino } \\
\text { Genicity }\end{array}$ & $\begin{array}{l}\text { Immuno } \\
\text { Toxicity }\end{array}$ & $\begin{array}{l}\text { Muta } \\
\text { Genicity }\end{array}$ & $\begin{array}{l}\text { Cyto } \\
\text { Toxicity }\end{array}$ & $\begin{array}{l}\text { LD50 } \\
(\mathrm{mg} / \mathrm{kg})\end{array}$ & TC \\
\hline \multicolumn{17}{|c|}{ B.1.617.2. S-Protein-NTD Mutant Type } \\
\hline $\begin{array}{l}\text { Standard } \\
\text { Drug }\end{array}$ & Ceftriaxone & -1.68 & 214.98 & 36 & 15 & 5 & 2 & 0.9477 & 1.486 & $0.55^{\text {(mod) }}$ & $0.51^{\text {(mod) }}$ & $0.99^{(-)}$ & $0.68^{\text {(mild) }}$ & $0.66^{\text {(mild) }}$ & 10,000 & VI \\
\hline \multirow{9}{*}{ H. cordata } & $\begin{array}{l}\text { Cholest-4,14-dien- } \\
\text { 15,20-diol-3,16- } \\
\text { dione }\end{array}$ & 4.87 & 74.60 & 31 & 4 & 2 & 0 & 0.9931 & 4.137 & $0.87^{(-)}$ & $0.62^{\text {(mild) }}$ & $0.70^{(+)}$ & $0.77^{(-)}$ & $0.66^{(-)}$ & 5000 & $\mathrm{~V}$ \\
\hline & Dihydrocelastrol & 6.15 & 77.75 & 33 & 4 & 3 & 1 & 0.9905 & 2.415 & $0.63^{\text {(mild) }}$ & $0.51^{\text {(mod) }}$ & $0.73^{(+)}$ & $0.88^{(-)}$ & $0.84^{(-)}$ & 1000 & IV \\
\hline & Naltrindole & 3.80 & 68.72 & 31 & 5 & 3 & 0 & 0.9848 & 4.214 & $0.89^{(-)}$ & $0.58^{\text {(mod) }}$ & $0.96^{(-)}$ & $0.57^{\text {(mod) }}$ & $0.55^{\text {(mod) }}$ & 402 & IV \\
\hline & Pirenperone & 3.49 & 54.69 & 29 & 5 & 0 & 0 & 0.9896 & 2.368 & $0.78^{(-)}$ & $0.63^{\text {(mild) }}$ & $0.99^{(-)}$ & $0.54^{(\bmod )}$ & $0.68^{\text {(mild) }}$ & 1000 & IV \\
\hline & Quercitrin & 1.68 & 131.35 & 22 & 7 & 5 & 0 & 0.9833 & 2.559 & $0.69^{\text {(mild) }}$ & $0.68^{\text {(mild) }}$ & $0.87^{(-)}$ & $0.51^{(\bmod )}$ & $0.99^{(-)}$ & 159 & III \\
\hline & Rhodoxanthin & 9.29 & 34.14 & 42 & 2 & 0 & 2 & 0.9902 & 2.26 & $0.63^{\text {(mild) }}$ & $0.61^{\text {(mild) }}$ & $0.60^{\text {(mild) }}$ & $0.90^{(-)}$ & $0.83^{(-)}$ & 10,000 & VI \\
\hline & Sesamin & 3.69 & 55.40 & 26 & 6 & 0 & 0 & 0.9871 & 0.967 & $0.81^{(-)}$ & $0.65^{\text {(mild) }}$ & $0.84^{(+)}$ & $0.60^{\text {(mild) }}$ & $0.94^{(-)}$ & 1500 & III \\
\hline & Usambarensine & 6.17 & 47.71 & 33 & 4 & 2 & 1 & 0.9970 & 2.689 & $0.91^{(-)}$ & $0.71^{(-)}$ & $0.86^{(+)}$ & $0.50^{\text {(mod) }}$ & $0.66^{\text {(mild) }}$ & 370 & IV \\
\hline & Biflorin & -0.70 & 160.81 & 25 & 9 & 6 & 1 & 0.9009 & 2.995 & $0.81^{(-)}$ & $0.78^{(-)}$ & $0.81^{(-)}$ & $0.51^{\text {(mod) }}$ & $0.83^{(-)}$ & 562 & IV \\
\hline \multirow{3}{*}{ S. aromaticum } & Crategolic acid & 5.81 & 77.75 & 34 & 4 & 3 & 1 & 0.9643 & 2.316 & $0.65^{\text {(mild) }}$ & $0.63^{\text {(mild) }}$ & $0.61^{\text {(mild) }}$ & $0.87^{(-)}$ & $0.89^{(-)}$ & 2000 & IV \\
\hline & Oleanolic acid & 6.72 & 57.53 & 33 & 3 & 2 & 1 & 0.9853 & 2.034 & $0.52^{\text {(mod) }}$ & $0.57^{(\mathrm{mod})}$ & $0.79^{(+)}$ & $0.85^{(-)}$ & $0.99^{(-)}$ & 2000 & IV \\
\hline & Rhamnetin & 2.22 & 120.36 & 23 & 7 & 4 & 0 & 0.9840 & 2.542 & $0.73^{\text {(mild) }}$ & $0.59^{\text {(mod) }}$ & $0.55^{\text {(mod) }}$ & $0.69^{\text {(mild) }}$ & $0.91^{(-)}$ & 5000 & $\mathrm{~V}$ \\
\hline \multicolumn{17}{|c|}{ Kabasura kudineer } \\
\hline \multirow{3}{*}{ C. serratum } & Acteoside & -0.45 & 245.29 & 44 & 15 & 9 & 3 & & & $0.81^{(-)}$ & $0.81^{(-)}$ & $0.99^{(+)}$ & $0.87^{(-)}$ & $0.77^{(-)}$ & 5000 & $\mathrm{~V}$ \\
\hline & Serratagenic acid & 5.43 & 94.83 & 35 & 5 & 3 & 1 & 0.9853 & 2.233 & $0.69^{\text {(mild) }}$ & $0.55^{\text {(mod) }}$ & $0.79^{(+)}$ & $0.90^{(-)}$ & $0.91^{(-)}$ & 6176 & VI \\
\hline & Verbascoside & -0.45 & 245.29 & 44 & 15 & 9 & 3 & 0.6642 & 2.694 & $0.81^{(-)}$ & $0.81^{(-)}$ & $0.99^{(+)}$ & $0.87^{(-)}$ & $0.77^{(-)}$ & 5000 & $\mathrm{~V}$ \\
\hline H. auriculata & Cucurbitacin B & 2.83 & 138.20 & 40 & 8 & 3 & 1 & 0.9895 & 4.041 & $0.87^{(-)}$ & $0.50^{\text {(mod) }}$ & $0.90^{(+)}$ & $0.72^{(-)}$ & $0.66^{\text {(mild) }}$ & 14 & II \\
\hline A. paniculata & Neoandrographolide & 1.17 & 125.69 & 34 & 8 & 4 & 0 & 0.8124 & 3.165 & $0.92^{(-)}$ & $0.88^{(-)}$ & $0.97^{(+)}$ & $0.69^{(\mathrm{mod})}$ & $0.70^{(+)}$ & 5 & I \\
\hline \multirow{2}{*}{ M. cerviana } & Orientin & 0.03 & 201.27 & 32 & 11 & 8 & 2 & 0.8864 & 3.207 & $0.81^{(-)}$ & $0.72^{(-)}$ & $0.52^{\text {(mod) }}$ & $0.52^{\text {(mod) }}$ & $0.87^{(-)}$ & 1213 & IV \\
\hline & Vitexin & 0.52 & 181.04 & 31 & 10 & 7 & 1 & 0.8984 & 2.724 & $0.81^{(-)}$ & $0.72^{(-)}$ & $0.82^{(-)}$ & $0.52^{\text {(mod) }}$ & $0.87^{(-)}$ & 832 & IV \\
\hline T. cordifolia & Tinosporide & 2.02 & 98.51 & 27 & 7 & 1 & $\begin{array}{l}1 \\
0\end{array}$ & $\begin{array}{l}.0904 \\
0.9589\end{array}$ & 2.775 & $0.82^{(-)}$ & $0.6^{\text {(mild) }}$ & $0.96^{(+)}$ & $0.72^{(-)}$ & $0.53^{(\mathrm{mod})}$ & $\begin{array}{l}032 \\
280\end{array}$ & III \\
\hline
\end{tabular}

e swallowed $(300<$ LD50 $\leq 2000)$; Class V: may be harmful if swallowed (2000 < LD50 $\leq 5000)$; Class VI: non-toxic (LD50 > 5000). 
Table 5. Identification of drug-likeness and toxicity analysis for selected compounds inhibits RBD-domain in S-Protein.

\begin{tabular}{|c|c|c|c|c|c|c|c|c|c|c|c|c|c|c|c|c|}
\hline \multicolumn{2}{|c|}{ Phytocompounds } & \multicolumn{6}{|c|}{ Drug-Likeness } & \multicolumn{9}{|c|}{ Toxicity Analysis } \\
\hline Plant Name & Compound Name & $\operatorname{miLog} \mathrm{P}$ & TPSA & Natoms & nON & nOHNH & $\begin{array}{l}\text { No. of } \\
\text { Violations }\end{array}$ & $\begin{array}{l}\text { Intestinal } \\
\text { Absorption }\end{array}$ & $\begin{array}{l}\text { Oral Toxicity } \\
\text { (-log kg/mol) }\end{array}$ & $\begin{array}{l}\text { Hepato } \\
\text { Toxicity }\end{array}$ & $\begin{array}{l}\text { Carcino } \\
\text { Genicity }\end{array}$ & $\begin{array}{l}\text { Immuno } \\
\text { Toxicity }\end{array}$ & $\begin{array}{l}\text { Muta } \\
\text { Genicity }\end{array}$ & $\begin{array}{l}\text { Cyto } \\
\text { Toxicity }\end{array}$ & $\begin{array}{l}\text { LD50 } \\
(\mathrm{mg} / \mathrm{kg})\end{array}$ & TC \\
\hline \multicolumn{17}{|c|}{ B.1.617.2. S-Protein-RBD Domain } \\
\hline \multirow{7}{*}{ H. cordata } & $\begin{array}{l}\text { 15,20-diol-3,16- } \\
\text { dione }\end{array}$ & 4.87 & 74.60 & 31 & 4 & 2 & 0 & 0.9931 & 4.137 & $0.87^{(-)}$ & $0.62^{\text {(mild) }}$ & $0.70^{(+)}$ & $0.77^{(-)}$ & $0.66^{(-)}$ & 5000 & $\mathrm{~V}$ \\
\hline & $\begin{array}{l}\text { Fluorometholone } \\
\text { 17-acetate }\end{array}$ & 3.09 & 80.67 & 30 & 5 & 1 & 0 & 0.6371 & 3.218 & $0.86^{(-)}$ & $0.61^{\text {(mod) }}$ & $0.99^{(-)}$ & $0.94^{(-)}$ & $0.71^{\text {(mild) }}$ & 4000 & $\mathrm{~V}$ \\
\hline & Progeldanamycin & 2.47 & 108.25 & 34 & 7 & 4 & 0 & 0.5100 & 3.001 & $0.63^{(\mathrm{mod})}$ & $0.59^{(\bmod )}$ & $0.99^{(-)}$ & $0.719^{\text {(mild) }}$ & $0.70^{\text {(mild) }}$ & 1000 & IV \\
\hline & Quercetin & 1.68 & 131.35 & 22 & 7 & 5 & 0 & 0.4381 & 2.636 & $0.69^{(\bmod )}$ & $0.68^{(\bmod )}$ & $0.87^{(-)}$ & $0.51^{(+)}$ & $0.99^{(-)}$ & 159 & III \\
\hline & Rhodoxanthin & 9.29 & 34.14 & 42 & 2 & 0 & 2 & 0.8190 & 2.660 & $0.63^{(\mathrm{mod})}$ & $0.61^{\text {(mod) }}$ & $0.60^{(\mathrm{mod})}$ & $0.90^{(-)}$ & $0.83^{(-)}$ & 10,000 & VI \\
\hline & $\begin{array}{l}\text { Stigmastane-3,6- } \\
\text { dione, } \\
\text { (5.alpha.) }\end{array}$ & 7.76 & 34.14 & 31 & 2 & 0 & 1 & 0.8690 & 2.962 & $0.78^{\text {(mild) }}$ & $0.62^{\text {(mod) }}$ & $0.99^{(-)}$ & $0.93^{(-)}$ & $0.58^{(\mathrm{mod})}$ & 775 & IV \\
\hline & Rhamnetin & 2.22 & 120.36 & 23 & 7 & 4 & 0 & 0.5620 & 2.739 & $0.73^{\text {(mild) }}$ & $0.59^{(\mathrm{mod})}$ & $0.55^{(\mathrm{mod})}$ & $0.69^{(\mathrm{mod})}$ & $0.91^{(-)}$ & 5000 & V \\
\hline \multirow{2}{*}{$\begin{array}{l}\text { Mitragyna } \\
\text { speciosa Korthi }\end{array}$} & Beta-Sitosterol & 8.62 & 20.23 & 30 & 1 & 1 & 1 & 0.9241 & 3.181 & $0.87^{(-)}$ & $0.60^{(\bmod )}$ & $0.99^{(+)}$ & $0.98^{(-)}$ & $0.94^{(-)}$ & 890 & IV \\
\hline & Stigmasterol & 7.87 & 20.23 & 30 & 1 & 1 & 1 & 0.9241 & 3.251 & $0.87^{(-)}$ & $0.60^{(\mathrm{mod})}$ & $0.99^{(+)}$ & $0.98^{(-)}$ & $0.94^{(-)}$ & 890 & IV \\
\hline \multicolumn{17}{|c|}{ Kabasura kudineer } \\
\hline T. cordifolia & Berberine & 0.20 & 40.82 & 25 & 5 & 0 & 0 & 0.4693 & 2.785 & $0.82^{(-)}$ & $0.56^{\text {(mod) }}$ & $0.99^{(-)}$ & $0.62^{\text {(mod) }}$ & $0.96^{(-)}$ & 200 & III \\
\hline C. serratum & Clerodermic acid & 2.73 & 63.60 & 24 & 4 & 1 & 0 & 0.7051 & 3.101 & $0.82^{(-)}$ & $0.55^{\text {(mod) }}$ & $0.81^{(-)}$ & $0.89^{(-)}$ & $0.80^{(-)}$ & 3300 & V \\
\hline C. speciosus & Diosgenin & 5.93 & 38.70 & 30 & 3 & 1 & 1 & 0.7820 & 3.364 & $0.69^{(\mathrm{mod})}$ & $0.55^{(\mathrm{mod})}$ & $0.99^{(-)}$ & $0.96^{--}$ & $0.99^{(-)}$ & 8000 & VI \\
\hline
\end{tabular}

Note: TC-Toxicity Class; Class I: fatal if swallowed (LD50 $\leq 5)$; Class II: fatal if swallowed ( $<$ LD50 $\leq 50)$; Class III: toxic if 
We finally identified the compounds acteoside and verbascoside from C. serratum as acting effectively against the mutated NTD (Figure 4D-I,K,L). The acteoside demonstrated more beneficial interactions with PHE86, ASN87, ASP88, ASN196, ASP198, GLY199, ILE233, and GLN234 via hydrogen bonds with a binding affinity of $-7.3 \mathrm{kcal} / \mathrm{mol}$. It also holds alkyl interactions with the ILE197. It is surrounded by hydrophobic residues (LEU54, LYS195, THR236). The toxicity class was also identified as V. The verbascoside interacts with ASN87, ASP88*, ASN196, ASP198, GLY199, ILE233, and GLN234 via carbon-hydrogen* bond with a binding affinity of $-7.1 \mathrm{kcal} / \mathrm{mol}$. It also held pi-alkyl interaction with LEU54 and PRO272, as well as pi-sigma bonding with THR236, and was surrounded by the hydrophobic residues (GLN52, PRO85, and ILE235). The predicted toxicity class of verbascoside was V. The standard drug ceftriaxone interacted with ASP88, ASN87, GLN115, ASN156*, ASP198, GLY199, GLY232, GLN234*, and ILE233 by hydrogen, carbonhydrogen, and pi-alkyl bonds. It was also surrounded by hydrophobic residues ASN196 and ILE197, and the predicted toxicity class was VI. Even though the standard drug ceftriaxone interacted with the crucial residues GLN234* by carbon-hydrogen bond, the binding affinity was $-6.3 \mathrm{kcal} / \mathrm{mol}$.

Moreover, the compounds kanzonol V, progeldanamycin, and rhodoxanthin from $H$. cordata excellently inhibit the RBD domain compared with the other tested phytocompounds (Figure 5D-L,N-P). Kanzonol V strongly interacted with GLU484 and TYR449 by hydrogen bond and showed pi-alkyl interactions with PHE456 and TYR489. Kanzonol V is surrounded by hydrophobic residues LEU452, LEU455, PHE490, and LEU492. The binding affinity is $-6.8 \mathrm{kcal} / \mathrm{mol}$. It belonged to the toxicity classes of V. Progeldanamycin has hydrogen bond interactions with TYR449, and SER494, as well as pi-sigma interaction with LEU452, and is also surrounded by the hydrophobic residues ASN450, PHE490, LEU492, and GLN493. The observed binding affinity is $-6.4 \mathrm{kcal} / \mathrm{mol}$ with the toxicity class IV. Rhodoxanthin shows pi-alkyl interactions with LEU452, TYR489, and PHE490 and was surrounded by the hydrophobic residues ARG346, SER349, TYR351, ASN450, LEU455, PHE456, GLU484, GLY485, PHE486, LEU492, GLN493, and SER494 with the binding affinity of $-7.5 \mathrm{kcal} / \mathrm{mol}$. The toxicity class was noticed as VI. However, the ceftriaxone did not interact with the specifically defined residues L452R, T478K, and E484Q in the Indian delta variant, and the binding affinity was observed as $-6.5 \mathrm{kcal} / \mathrm{mol}$.

\section{Ceftriaxone}

A)

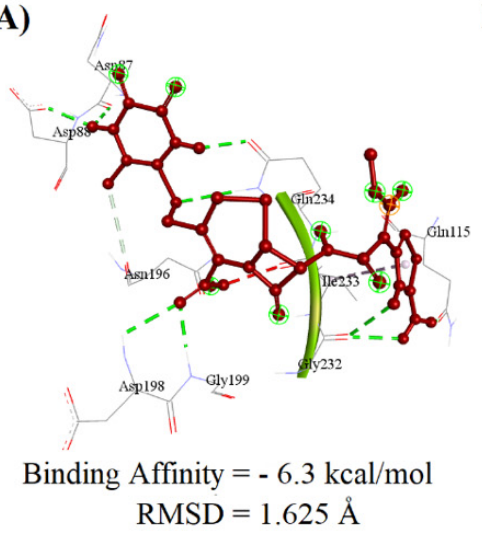

B)

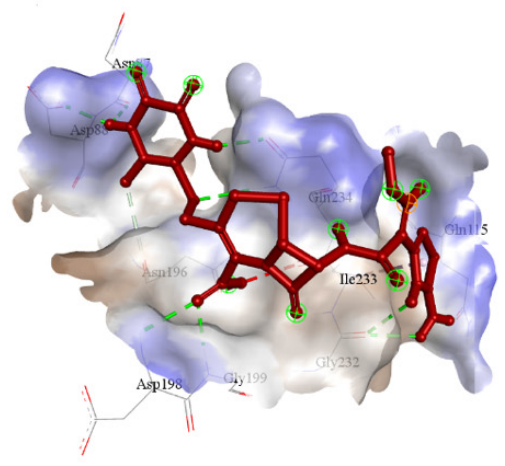

C)

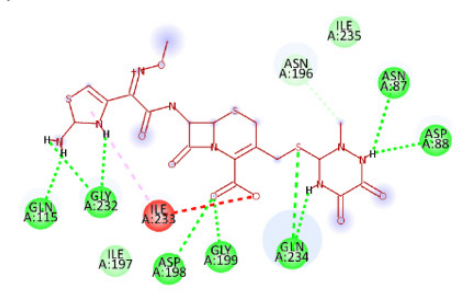

\section{Acetoside}

Figure 4. Cont. 


\section{Acetoside}

D)

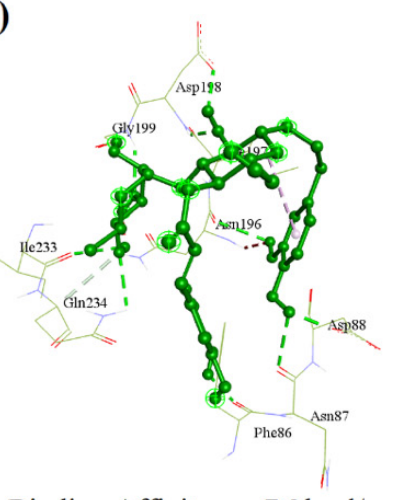

Binding Affinity $=-7.3 \mathrm{kcal} / \mathrm{mol}$ $\mathrm{RMSD}=2.804 \AA$

G)

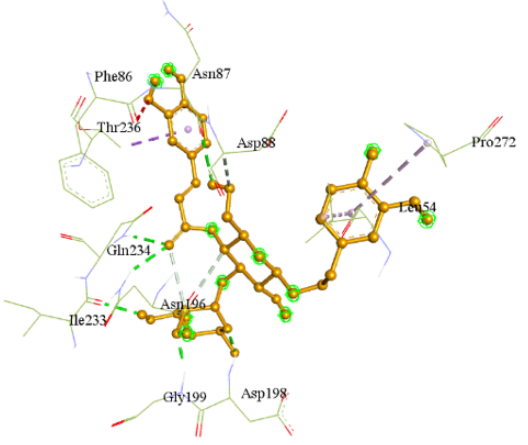

Binding Affinity $=-7.1 \mathrm{kcal} / \mathrm{mol}$ $\operatorname{RMSD}=1.904 \AA$
E)

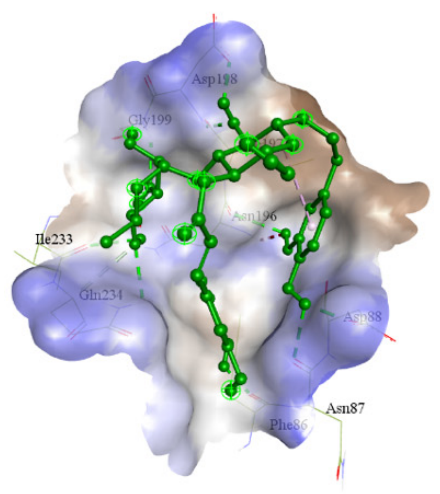

\section{Verbascoside}

H)

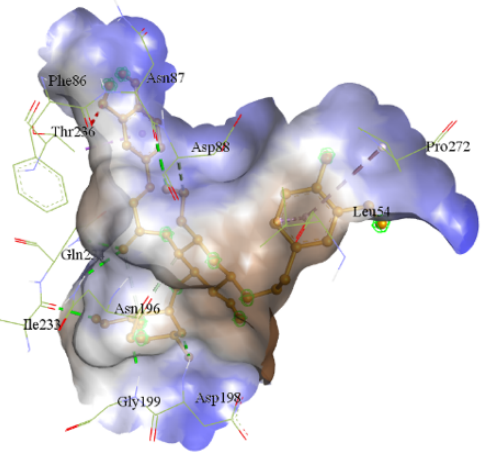

F)

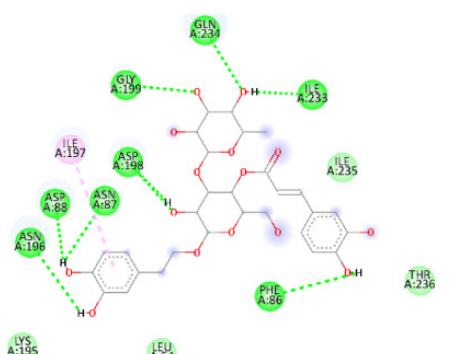

A.195
I)

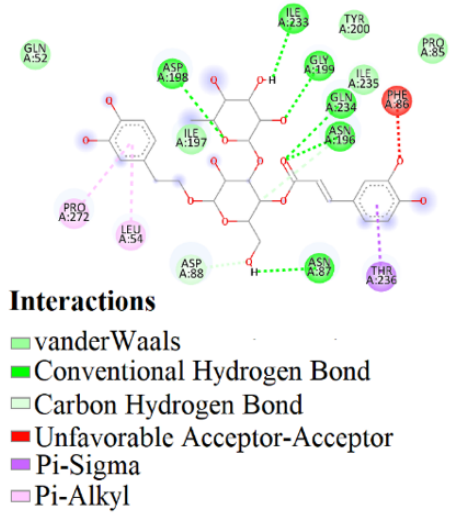

J)

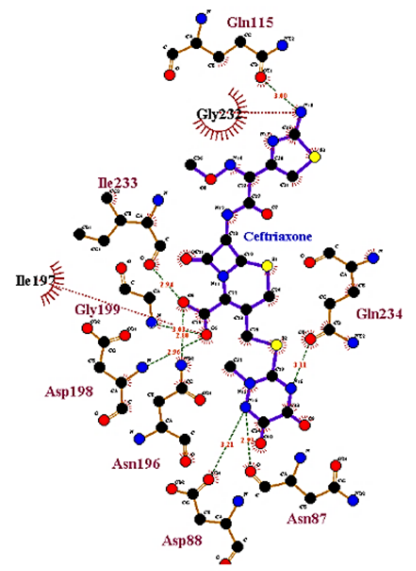

K)

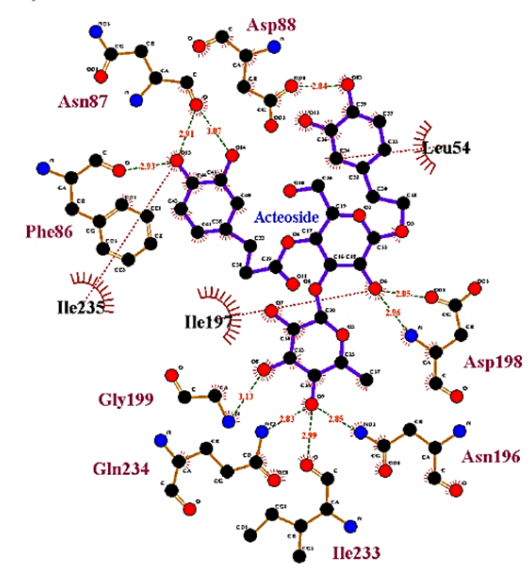

L)

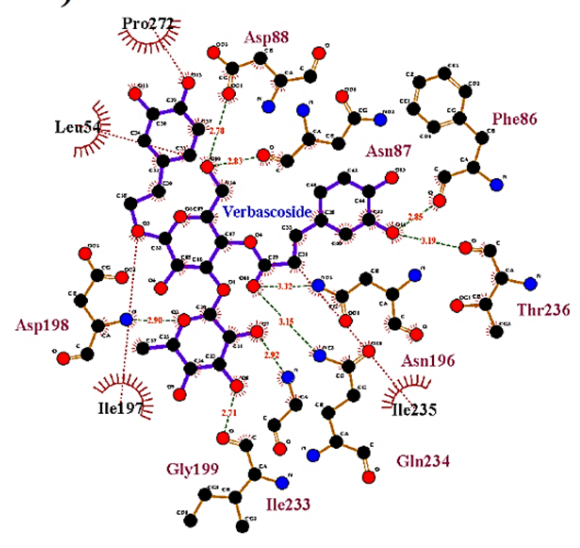

Figure 4. The docking pose of the mutated NTD with the most effective phytocompounds is based on the binding affinity and interacting residues $(\mathbf{A}, \mathbf{D}, \mathbf{G})$. The docking poses with ceftriaxone, aceteoside, and verbascoside, respectively $(\mathbf{B}, \mathbf{E}, \mathbf{H})$. The hydrophobicity of the interacting residues (brown ( $\uparrow$ hydrophobicity)-blue ( $\downarrow$ hydrophobicity), (C,F,I). The type of bonds involved in interacting phytocompounds with the mutated NTD residues $(\mathbf{J}-\mathbf{L})$. The Ligplot interaction for the phytocompounds docked with the mutated NTD residues. 


\section{Ceftriaxone}

A)

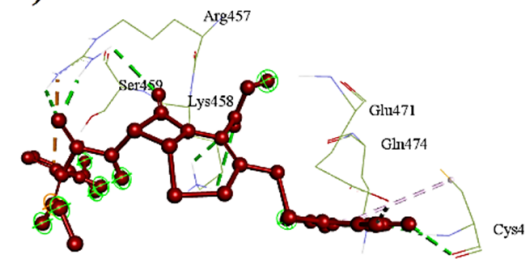

Binding Affinity $=-6.5 \mathrm{kcal} / \mathrm{mol}$ $\mathrm{RMSD}=1.875 \AA$

D)

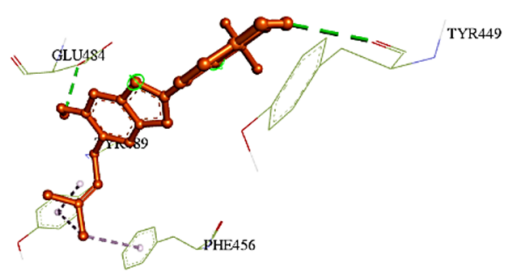

Binding Affinity $=-6.8 \mathrm{kcal} / \mathrm{mol}$ $\mathrm{RMSD}=2.182 \AA$

G)

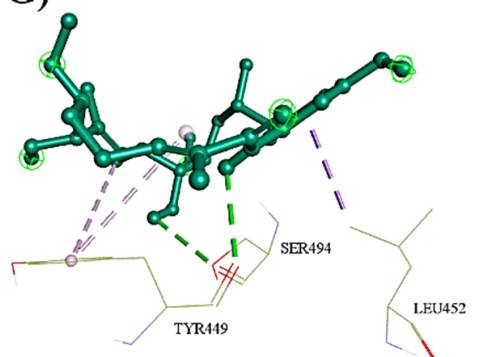

Binding Affinity $=-6.4 \mathrm{kcal} / \mathrm{mol}$ $\mathrm{RMSD}=2.392 \AA$

J)

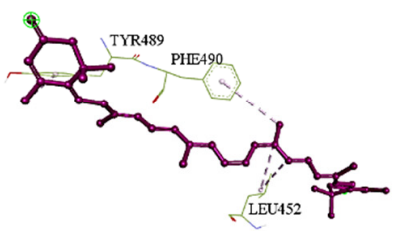

Binding Affinity $=-7.5 \mathrm{kcal} / \mathrm{mol}$ $\mathrm{RMSD}=1.856$

B)

E)

K)

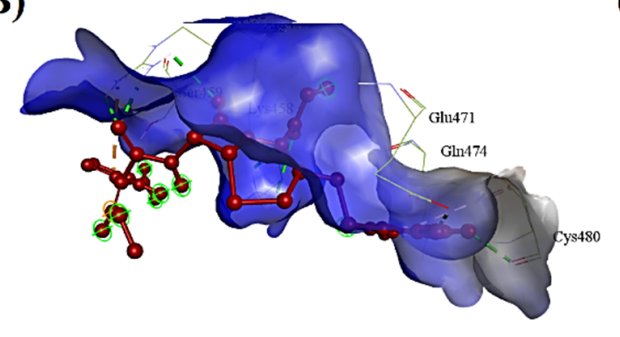

Kanzonol V

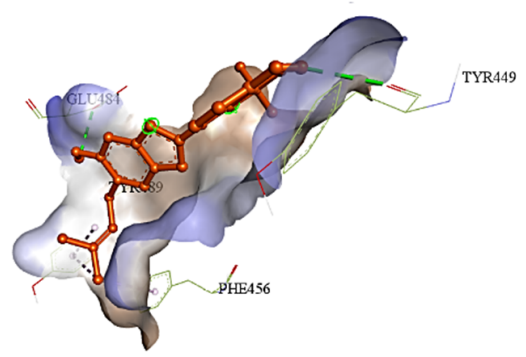

Progeldanamycin

H)

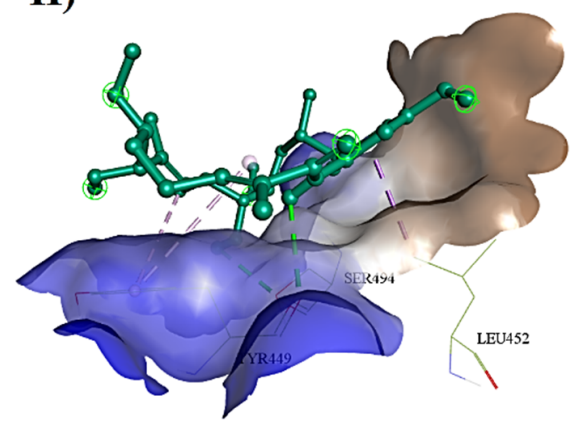

Rhodoxanthin

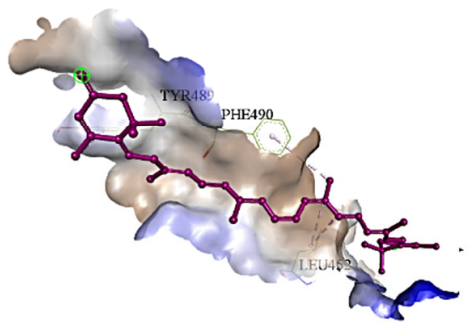

C)

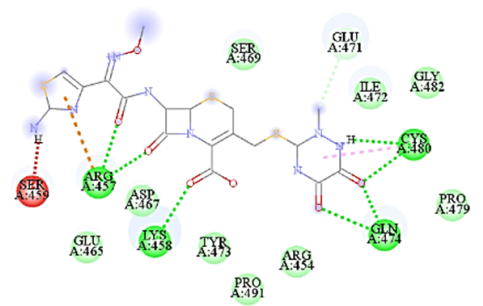

F)

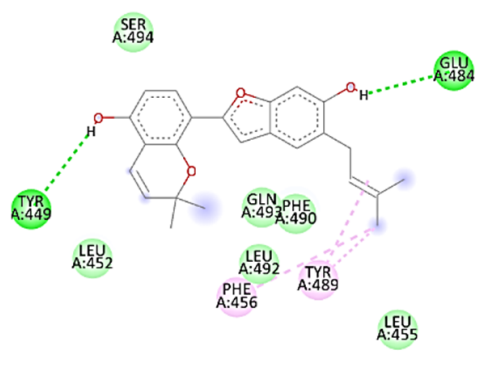

I)

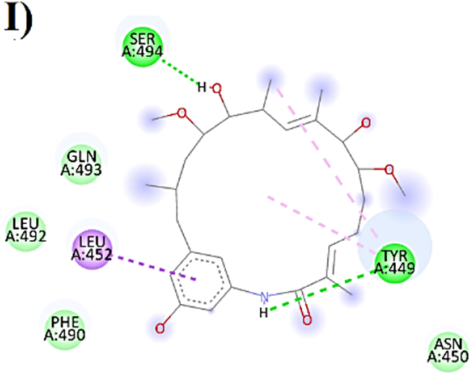

L)

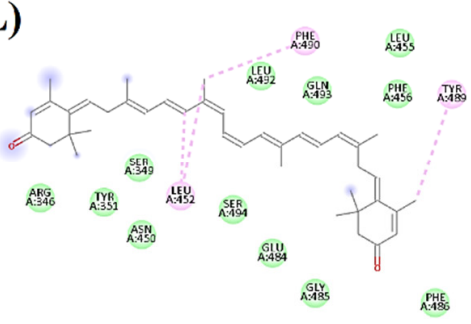

Interactions

$\square$ van der Waals $\square$ Alkyl $\square$ Pi-Sigma

$\square$ Pi-cation $\quad \square$ Pi-Alkyl

- Conventional Hydrogen Bond

$\square$ Carbon Hydrogen Bond

Figure 5. Cont. 
M)

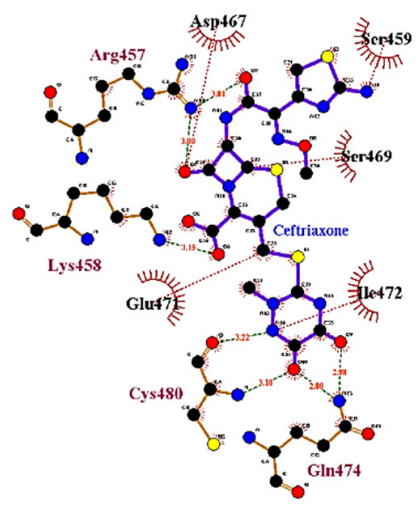

N)

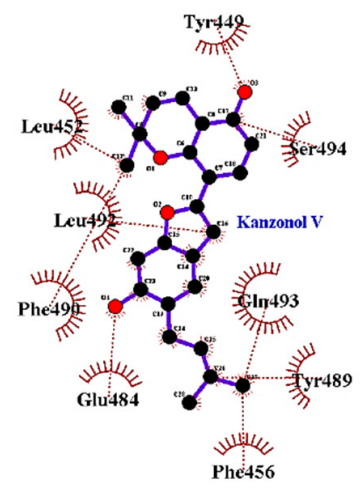

O)

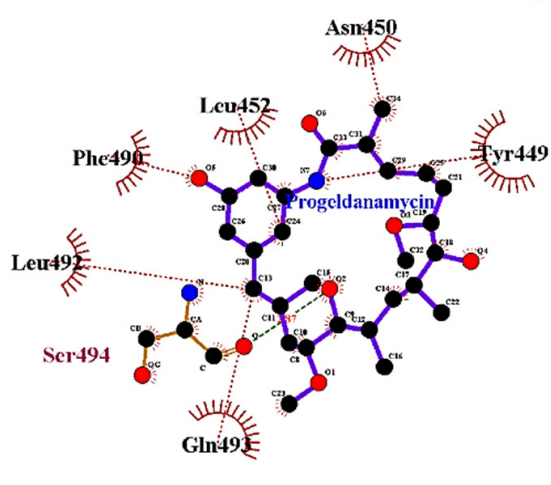

P)

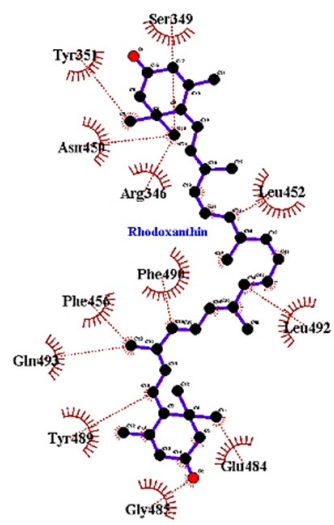

Figure 5. The docking pose of the RBD with the most promising phytocompounds based on the binding affinity and interacting residues $(\mathbf{A}, \mathbf{D}, \mathbf{G}, \mathbf{J})$. The docking poses with ceftriaxone, kanzonol $\mathrm{V}$, progeldanamycin and rhodoxanthin $(\mathbf{B}, \mathbf{E}, \mathbf{H}, \mathbf{K})$. The hydrophobicity of the interacting residues (brown ( $\uparrow$ hydrophobicity)-blue ( $\downarrow$ hydrophobicity), (C,F,I,L). The type of bonds involved in interacting phytocompounds with RBD residues (M-P). The LigPlot interaction for the phytocompounds docked with RBD residues.

\section{Discussion}

The altered viral subset will become predominant in the context of environmental switches. In the face of such problems in treatment planning, there is now strong necessity for alternative therapy. Natural substances, which have been used globally for many years due to their chemical variety, species diversity, and drug-like features, may be recognized in this category. In this line, we aimed to assess the anti-COVID-19 capability of Siddha polyherbal formulation and plant extracts containing different phytocompounds that have been previously reported to have antiviral properties $[54,55]$. The SARS-spike CoV-2 protein has undergone mutations and is heavily glycosylated; hence, the biological relevance of viral alterations must be investigated promptly $[40,131]$. Li et al. examined over 80 variations and 26 glycosylation site variations to identify the severity of disease transmission and sensitivity from recovered patients. The mutated residue N234Q was significantly resistant to neutralizing antibodies, whereas N165Q became more susceptible [48]. Similarly, the Indian delta variant B.1.617.2. carries five particular mutations: L452R, T478K, E484Q, D614G, and P681R [12,20,132]. It controls viral fitness by increasing the ACE2 receptor binding affinity, increasing infectivity, and deactivating the antibodies. These mutations in the Indian delta variant increased the spike's stability, viral infectivity, and stronger cell attachment, thereby promoting viral replication, transmissibility, and pathogenicity $[12,29,133,134]$. These discoveries could help with vaccine and therapeutic antibody development [40].

Drugs derived from medicinal plants have traditionally been widely utilized to treat diseases [135-138]. However, effective medication is strongly advocated at this key stage of COVID-19 and its variant infection [1]. Plants are rich in phytocompounds, which could efficiently counteract with COVID-19 [139,140]. Hence, the MT-NTD and RBD domains in the SARS-CoV-2 were docked using 603 phytochemicals in this investigation. Many of the phytocompounds found in plants had high protein-binding abilities. After applying the threshold criteria of $\leq-6.0 \mathrm{kcal} / \mathrm{mol}$ to 603 compounds, 27 and 13 phytocompounds for the MT-NTD and RBD, respectively, were screened.

Phytocompounds with a binding affinity of $\leq-6.0 \mathrm{kcal} / \mathrm{mol}$ were chosen because they showed promising inhibition with crucial residues N234Q, N165Q, L452R, T478K, and E484Q against the MT-NTD and RBD. Typically, the threshold is chosen by correlating it to known inhibitors that have been previously published and biologically verified. In this regard, we selected ceftriaxone, a standard drug that evidenced higher binding affinity toward both the NTD and RBD than the FDA-approved anti-viral drugs lopinavir [141], 
remdesivir [142], chloroquine [143], umifenovir [144], favipiravir [145], ribavirin [146], hydroxychloroquine [147], sofosbuvir [148], and oseltamivir [4]. However, ceftriaxone shows less binding affinity at $-6.3 \mathrm{kcal} / \mathrm{mol}$ and $-6.5 \mathrm{kcal} / \mathrm{mol}$ towards the MT-NTD and RBD domains. Likewise, there is no interaction with the specified residues accounting for the targeted MT-NTD and RBD of the S-Protein of the Indian delta variant B.1.617.2.

The root of $C$. serratum is reported to treat respiratory disease, fever, inflammation, cancer, nociceptive, liver disorders, and allergic condition, as well as to act as an antioxidant $[63,64,116,117]$. Acteoside and verbascoside from C. serratum act well against the MT-NTD. Acteoside holds eight hydrogen bonds, one alkyl, and three hydrophobic interactions with $-7.3 \mathrm{kcal} / \mathrm{mol}$ of binding affinity. Moreover, verbascoside shows seven hydrogen bonds, two alkyl bonds, one pi-sigma bond, and three hydrophobic interactions with the binding affinity of $-7.1 \mathrm{kcal} / \mathrm{mol}$. These two phytocompounds have specifically interacted with the mutated residues GLN234. Kallingal et al. reported that acteoside from Tectona grandis acts effectively against SARS-CoV-2 proteases [149]. Shawky et al. reported that verbascoside from Cichorium intybus, Olea europaea, and Marrubium vulgare acts as a potent anti-COVID-19 compound [150]. H. cordata is implemented to treat many diseases related to the lungs, especially against viruses such the pseudorabies herpes virus ( $P r V)$, human noroviruses (HuNoVs), murine coronaviruses, and the dengue virus infection; regulate innate immune modulation activities positively, and also inhibit the replication of SARS-CoV [65,91-98].

Nevertheless, the ceftriaxone constitutes less binding affinity $-6.5 \mathrm{kcal} / \mathrm{mol}$, and it does not interact with the targeted residues L452R, T478K, and E484Q in the RBD. The compounds kanzonol V $(-6.8 \mathrm{kcal} / \mathrm{mol})$, progeldanamycin $(-6.4 \mathrm{kcal} / \mathrm{mol})$, and rhodoxanthin $(-7.5 \mathrm{kcal} / \mathrm{mol})$ from $\mathrm{H}$. cordata act effectively against the target residues in the RBD of the Indian delta variant B.1.617.2 with better binding affinity. Even though kanzonol and rhodoxanthin violated one or two Lipinski rules, they beneficially treated bacterial and fungal infections, as previously reported [151]. Additionally, the toxicity class is satisfactorily categorized as V and VI for kanzonol V and rhodoxanthin. Progeldanamycin is not violating any rules, and its toxicity class is described as VI. In addition, Benet et al. explained that violation of two or more Lipinski rules for the natural products and their derivatives is acceptable. However, the FDA-approved oral drugs under the category Class 1 (acarbose, cyanocobalamin, everolimus, ivermectin, etc.) are believed to have high solubility properties and high permeability, also violating the Lipinski rule [152].

Moreover, the maximum number of phytocompounds from $H$. cordat $a$ and Kabasura kudineer act significantly well against the NTD and RBD domain of the Indian delta variant B.1.617.2 S-protein. AYUSH has recommended many treatments towards COVID-19 prevention, which are implemented as preventative and symptomatic therapy in COVID-19 management $[153,154]$. Nevertheless, no Siddha formulation was prescribed for COVID-19 containing H. cordata, including Kabasura kudineer and Ayush kwath, which has already been recommended by the Indian government $[60,155]$. According to the current investigation, the formulations and phytocompounds examined in this study showed considerably greater binding efficacy against the MT-NTD and RBD in the S-Protein of the Indian delta variant B.1.617.2. In silico studies suggested that the resulting phytocompounds may operate as efficient inhibitors of the Indian delta variant B.1.617.2 by binding to the spike glycoprotein, which may be investigated further in vitro to develop improved herbal formulations and anti-viral drugs. Furthermore, SARS-CoV-2 has been proven to have a greater affinity for pharyngeal epithelial cells [55]. Since these extracts can be delivered to the pharyngeal regions through appropriate oral formulations, they will be effective to control the infection rates. 


\section{Conclusions}

During the COVID-19 disease outbreak caused by SARS-CoV-19 and its variants the disease transmission heightened due to a lack of targeted medications and vaccines. Even though vaccines have been identified, their efficacy against the Indian delta variant B.1.617.2 has dramatically decreased, forcing researchers to look for novel anti-viral formulations. In this regard, we analyzed 603 compounds from 22 plants. We identified five compounds: acteoside, verbascoside, kanzonol V, progeldanamycin, and rhodoxanthin, which acted significantly against the Indian delta variant B.1.617.2 compared with ceftriaxone, which is the most beneficial drug in COVID 19 treatment. Though the Siddha formulation contains C. serratum (L.) Moon, there is no Siddha formulation containing H. cordata. Hence, this study contributes to the evidence for developing pharmaceutical formulations and antiviral drugs that act specifically against the Indian delta variant B.1.617.2.

Supplementary Materials: The following are available online at https:/ / www.mdpi.com/article/ 10.3390/app12020665/s1, Figure S1: The sequence alignment for the wild- and mutated-type NTD in S-Protein; Figure S2: The LigPlot interaction for the mutated NTD domain and the screened phytocompounds based on the binding affinity $\leq-6.0 \mathrm{kcal} / \mathrm{mol}$; Figure S3: The LigPlot interaction for the RBD domain and the screened phytocompounds based on the binding affinity $\leq-6.0 \mathrm{kcal} / \mathrm{mol}$. Table S1: The molecular properties of screened phytocompounds with the $\leq-6.0 \mathrm{kcal} / \mathrm{mol}$ binding energy against mutated NTD Domain; Table S2: The molecular properties of screened phytocompounds with the $\leq-6.0 \mathrm{kcal} / \mathrm{mol}$ binding energy against RBD Domain; Table S3: The binding affinity, interacting residues, and bond length for the screened phytocompounds against the mutated NTD in the S-Protein; Table S4: The binding affinity, interacting residues, and bond length for the screened phytocompounds against the RBD in the S-Protein.

Author Contributions: Conceptualization, M.B., B.S.S., P.K., S.T. and C.C.; methodology, M.B.; software, M.B.; validation, M.B., B.S.S., S.T. and C.C; formal analysis, M.B.; investigation, M.B.; resources, C.C.; data curation, M.B.; writing—original draft preparation, M.B. and B.S.S.; writing—article and editing, M.B., B.S.S., P.K., S.T. and C.C.; supervision, C.C.; project administration, C.C. and B.S.S.; funding acquisition, C.C. All authors have read and agreed to the published version of the manuscript.

Funding: This research received no external funding.

Institutional Review Board Statement: Not applicable.

Informed Consent Statement: Not applicable.

Data Availability Statement: The data presented in this study are available within the article.

Acknowledgments: The authors gratefully acknowledge the Faculty of Pharmacy, Chiang Mai University, Chiang Mai, Thailand. The research was partially supported by Chiang Mai University, Chiang Mai, Thailand. B.M. thanks the CMU Presidential Scholarships for their kind support of her post-doctoral research.

Conflicts of Interest: The authors declare no conflict of interest.

\section{References}

1. Florindo, H.F.; Kleiner, R.; Vaskovich-Koubi, D.; Acúrcio, R.C.; Carreira, B.; Yeini, E.; Tiram, G.; Liubomirski, Y.; Satchi-Fainaro, R. Immune-mediated approaches against COVID-19. Nat. Nanotechnol. 2020, 15, 630-645. [CrossRef]

2. Rabaan, A.A.; Al-Ahmed, S.H.; Sah, R.; Tiwari, R.; Yatoo, M.I.; Patel, S.K.; Pathak, M.; Malik, Y.S.; Dhama, K.; Singh, K.P.; et al. SARS-CoV-2/COVID-19 and advances in developing potential therapeutics and vaccines to counter this emerging pandemic. Ann. Clin. Microbiol. Antimicrob. 2020, 19, 40. [CrossRef] [PubMed]

3. WHO Solidarity Trial Consortium; Pan, H.; Peto, R.; Henao-Restrepo, A.M.; Preziosi, M.P.; Sathiyamoorthy, V.; Karim, A.Q.; Alejandria, M.M.; García, H.C.; Kieny, M.P.; et al. Repurposed Antiviral Drugs for COVID-19—Interim WHO Solidarity Trial Results. N. Engl. J. Med. 2021, 384, 497-511. [PubMed]

4. Hu, X.; Zhou, Z.; Li, F.; Xiao, Y.; Wang, Z.; Xu, J.; Dong, F.; Zheng, H.; Yu, R. The study of anti-viral drugs targeting SARS-CoV-2 nucleocapsid and spike proteins through large-scale compound repurposing. Heliyon 2021, 7, e06387. [CrossRef] [PubMed]

5. Feldman, C.; Anderson, R. The role of co-infections and secondary infections in patients with COVID-19. Pneumonia (Nathan) 2021, 13, 5. [CrossRef] [PubMed] 
6. Nitulescu, G.M.; Paunescu, H.; Moschos, S.A.; Petrakis, D.; Nitulescu, G.; Ion, G.N.D.; Spandidos, D.A.; Nikolouzakis, T.K.; Drakoulis, N.; Tsatsakis, A. Comprehensive analysis of drugs to treat SARS-CoV-2 infection: Mechanistic insights into current COVID-19 therapies (Review). Int. J. Mol. Med. 2020, 46, 467-488. [CrossRef] [PubMed]

7. Iacob, S.; Iacob, D.G. SARS-CoV-2 Treatment Approaches: Numerous Options, No Certainty for a Versatile Virus. Front. Pharmacol. 2020, 11, 1224. [CrossRef]

8. Wu, C.; Liu, Y.; Yang, Y.; Zhang, P.; Zhong, W.; Wang, Y.; Wang, Q.; Xu, Y.; Li, M.; Li, X.; et al. Analysis of therapeutic targets for SARS-CoV-2 and discovery of potential drugs by computational methods. Acta Pharm. Sin B 2020, 10, 766-788. [CrossRef]

9. Wondmkun, Y.T.; Mohammed, O.A. A Review on Novel Drug Targets and Future Directions for COVID-19 Treatment. Biologics 2020, 14, 77-82. [CrossRef]

10. Faheem, B.K.; Sekhar, K.V.G.C.; Kunjiappan, S.; Jamalis, J.; Balaña-Fouce, R.; Tekwani, B.L.; Sankaranarayanan, M. Druggable targets of SARS-CoV-2 and treatment opportunities for COVID-19. Bioorg. Chem. 2020, 104, 104269. [CrossRef]

11. Eastman, R.T.; Roth, J.S.; Brimacombe, K.R.; Simeonov, A.; Shen, M.; Patnaik, S.; Hall, M.D. Remdesivir: A Review of Its Discovery and Development Leading to Emergency Use Authorization for Treatment of COVID-19. ACS Cent. Sci. 2020, 6, 672-683. [CrossRef]

12. Planas, D.; Veyer, D.; Baidaliuk, A.; Staropoli, I.; Guivel-Benhassine, F.; Rajah, M.M.; Planchais, C.; Porrot, F.; Robillard, N.; Puech, J. Reduced sensitivity of SARS-CoV-2 variant Delta to antibody neutralization. Nature 2021, 596, 276-280. [CrossRef]

13. Ye, S.; Zhang, Y.; Zhao, X.; Yu, Z.; Song, Y.; Tan, Z.; Tang, Y.; Chen, S.; Wang, M.; Ling, H. Emerging Variants of B.1.617 Lineage Identified Among Returning Chinese Employees Working in India-Chongqing Municipality, China, April 2021. China CDC Wkly. 2021, 3, 409-410. [CrossRef]

14. Liu, J.; Liu, Y.; Xia, H.; Zou, J.; Weaver, S.C.; Swanson, K.A.; Cai, H.; Cutler, M.; Cooper, D.; Muik, A.; et al. BNT162b2-elicited neutralization of B.1.617 and other SARS-CoV-2 variants. Nature 2021, 596, 273-275. [CrossRef] [PubMed]

15. Jacob, J.J.; Fletcher, J.G.; Priya, M.T.; Veeraraghavan, B.; Mutreja, A. Relevance of immune response and vaccination strategies of SARS-CoV-2 in the phase of viral red queen dynamics. Indian J. Med. Microbiol. 2021, 39, 417-422. [CrossRef] [PubMed]

16. Singh, J.; Pandit, P.; McArthur, A.G.; Banerjee, A.; Mossman, K. Evolutionary trajectory of SARS-CoV-2 and emerging variants. Virol. J. 2021, 18, 166

17. Singh, J.; Rahman, S.A.; Ehtesham, N.Z.; Hira, S.; Hasnain, S.E. SARS-CoV-2 variants of concern are emerging in India. Nat. Med. 2021, 27, 1131-1133. [CrossRef]

18. Hasan, M.S.; Islam, M.T.; Alam, A.S.M.R.U.; Sarkar, S.L.; Rahman, M.S.; Islam, O.K.; Setu, M.A.A.; Chakrovarty, T.; Al-Emran H.M.; Jahid, I.K.; et al. Initial reports of the SARS-CoV-2 Delta variant (B.1.617.2 lineage) in Bangladeshi patients: Risks of cross-border transmission from India. Health Sci. Rep. 2021, 4, e366. [CrossRef]

19. Winger, A.; Caspari, T. The Spike of Concern-The Novel Variants of SARS-CoV-2. Viruses 2021, 13, 1002. [CrossRef]

20. Mlcochova, P.; Kemp, S.; Dhar, M.S.; Papa, G.; Meng, B.; Ferreira, I.A.T.M.; Datir, R.; Collier, D.A.; Albecka, A.; Singh, S.; et al. Indian SARS-CoV-2 Genomics Consortium (INSACOG); Genotype to Phenotype Japan (G2P-Japan) Consortium; CITIID-NIHR BioResource COVID-19 Collaboration. SARS-CoV-2 B.1.617.2 Delta variant replication and immune evasion. Nature 2021, 599, 114-119. [CrossRef] [PubMed]

21. Worldometers. 2021. Available online: https:/ /www.worldometers.info/coronavirus / (accessed on 9 June 2021).

22. Schumaker, E. What to Kow about the Delta COVID-19 Variant First Detected in India. ABC News; 12 June 2021. Available online: https:/ / abcnews.go.com/Health/delta-covid-19-variant-detectedindia/story?id=78197319 (accessed on 12 July 2021).

23. European Centre for Disease Prevention and Control (ECDPC). SARS-CoV-2 Variants of Concern as of 7 October 2021. Available online: https:/ / cov-lineages.org/lineage.html?lineage=B.1.617.2 (accessed on 16 December 2021).

24. Mukherji, B.; Mcgregor, G. Countries Enact Travel Bans to Contain India's COVID Variant-And Protect against 'a Global Resurgence all over again. 2021. Available online: https:/ / fortune.com/2021/04/28/india-travel-ban-flight-ban-covid-variantcases-global-resurgence/ (accessed on 10 October 2021).

25. Phadnis, A. India Suspends UK Flights to Prevent the Spread of New COVID-19 Strain. Business Standard News. 2021. Available online: https:/ / www.business-standard.com/article/current-affairs/india-suspends-uk-flights-to-prevent-the-spread-of-newcovid-19-strain-120122100645_1.html (accessed on 10 October 2021).

26. Sanches, P.R.S.; Charlie-Silva, I.; Braz, H.L.B.; Bittar, C.; Calmon, F.M.; Rahal, P.; Cilli, E.M. Recent advances in SARS-CoV-2 Spike protein and RBD mutations comparison between new variants Alpha (B.1.1.7, United Kingdom), Beta (B.1.351, South Africa), Gamma (P.1, Brazil) and Delta (B.1.617.2, India). J. Virus Erad. 2021, 7, 100054. [CrossRef]

27. Yin, R.; Guest, J.D.; Taherzadeh, G.; Gowthaman, R.; Mittra, I.; Quackenbush, J.; Pierce, B.G. Structural and energetic profiling of SARS-CoV-2 receptor binding domain antibody recognition and the impact of circulating variants. PLoS Comput. Biol. 2021, 17, e1009380. [CrossRef]

28. Cherian, S.; Potdar, V.; Jadhav, S.; Yadav, P.; Gupta, N.; Das, M.; Rakshit, P.; Singh, S.; Abraham, P.; Panda, S.; et al. SARS-CoV-2 Spike Mutations, L452R, T478K, E484Q and P681R, in the Second Wave of COVID-19 in Maharashtra, India. Microorganisms 2021, 9, 1542. [CrossRef]

29. Cheng, M.H.; Krieger, J.M.; James, M.; Yufei, X.; Burak, K.; Yi, S.; Moshe, A.; Ivet, B. Impact of New Variants on SAR-CoV-2 Infectivity and Neutralization: A Molecular Assessment of the Alterations in the Spike-Host Protein Interactions. Lancet Preprints. Available online: https:/ / ssrn.com/abstract=3907841 (accessed on 10 October 2021).

30. WHO. Tracking SARS-CoV-2 Variants. 2021. Available online: https://www.who.int/en/activities/tracking-SARS-CoV-2variants / (accessed on 10 October 2021). 
31. Kirola, L. Genetic emergence of B.1.617.2 in COVID-19. New Microbes New Infect. 2021, 43, 100929. [CrossRef]

32. Wilhelm, A.; Toptan, T.; Pallas, C.; Wolf, T.; Goetsch, U.; Gottschalk, R.; Vehreschild, M.J.G.T.; Ciesek, S.; Widera, M. Antibody-Mediated Neutralization of Authentic SARS-CoV-2 B.1.617 Variants Harboring L452R and T478K/E484Q. Viruses 2021, 13, 1693. [CrossRef]

33. Ding, C.; He, J.; Zhang, X.; Jiang, C.; Sun, Y.; Zhang, Y.; Chen, Q.; He, H.; Li, W.; Xie, J.; et al. Crucial Mutations of Spike Protein on SARS-CoV-2 Evolved to Variant Strains Escaping Neutralization of Convalescent Plasmas and RBD-Specific Monoclonal Antibodies. Front. Immunol. 2021, 12, 693775. [CrossRef] [PubMed]

34. Deng, X.; Garcia-Knight, M.A.; Khalid, M.M.; Servellita, V.; Wang, C.; Morris, M.K.; Sotomayor-González, A.; Glasner, D.R.; Reyes, K.R.; Gliwa, A.S.; et al. Transmission, infectivity, and neutralization of a spike L452R SARS-CoV-2 variant. Cell 2021, 184, 3426-3437.e8. [CrossRef] [PubMed]

35. Plante, J.A.; Liu, Y.; Liu, J.; Xia, H.; Johnson, B.A.; Lokugamage, K.G.; Zhang, X.; Muruato, A.E.; Zou, J.; Fontes-Garfias, C.R.; et al. Spike mutation D614G alters SARS-CoV-2 fitness. Nature 2021, 592, 116-121. [CrossRef] [PubMed]

36. Salleh, M.Z.; Derrick, J.P.; Deris, Z.Z. Structural Evaluation of the Spike Glycoprotein Variants on SARS-CoV-2 Transmission and Immune Evasion. Int. J. Mol. Sci. 2021, 22, 7425. [CrossRef]

37. Jackson, C.B.; Farzan, M.; Chen, B.; Choe, H. Mechanisms of SARS-CoV-2 entry into cells. Nat. Rev. Mol. Cell Biol. 2021, 5, 1-18. [CrossRef] [PubMed]

38. Kaur, S.P.; Gupta, V. COVID-19 Vaccine: A comprehensive status report. Virus Res. 2020, 288, 198114. [CrossRef]

39. Dai, L.; Gao, G.F. Viral targets for vaccines against COVID-19. Nat. Rev. Immunol. 2021, 21, 73-82. [CrossRef]

40. Li, Y.D.; Chi, W.Y.; Su, J.H.; Ferrall, L.; Hung, C.F.; Wu, T.C. Coronavirus vaccine development: From SARS and MERS to COVID-19. J. Biomed. Sci. 2020, 27, 104. [CrossRef]

41. Anand, U.; Jakhmola, S.; Indari, O.; Jha, H.C.; Chen, Z.S.; Tripathi, V.; Lastra, P.J.M. Potential Therapeutic Targets and Vaccine Development for SARS-CoV-2/COVID-19 Pandemic Management: A Review on the Recent Update. Front. Immunol. 2021, 12, 658519. [CrossRef] [PubMed]

42. Li, Q.; Wu, J.; Nie, J.; Zhang, L.; Hao, H.; Liu, S.; Zhao, C.; Zhang, Q.; Liu, H.; Nie, L.; et al. The Impact of Mutations in SARS-CoV-2 Spike on Viral Infectivity and Antigenicity. Cells 2020, 182, 1284-1294.e9. [CrossRef]

43. Chung, J.Y.; Thone, M.N.; Kwon, Y.J. COVID-19 vaccines: The status and perspectives in delivery points of view. Adv. Drug Deliv. Rev. 2021, 170, 1-25. [CrossRef] [PubMed]

44. Cevik, M.; Grubaugh, N.D.; Iwasaki, A.; Openshaw, P. COVID-19 vaccines: Keeping pace with SARS-CoV-2 variants. Cell 2021, 184, 5077-5081. [CrossRef]

45. Koshy, J. Coronavirus I Single Dose Only 33\% Effective against B.1.617.2 Variant: U.K. 23 May 2021. The Hindu News. Available online: https:/ / www.thehindu.com/news/national/coronavirus-single-dose-only-33-effective-against-b16172-variant-uk/ article34628435.ece. (accessed on 10 October 2021).

46. Bernal, L.J.; Andrews, N.; Gower, C.; Gallagher, E.; Simmons, R.; Thelwall, S.; Stowe, J.; Tessier, E.; Groves, N.; Dabrera, G.; et al. Effectiveness of COVID-19 Vaccines against the B.1.617.2 (Delta) Variant. N. Engl. J. Med. 2021, 385, 585-594. [CrossRef] [PubMed]

47. Pollard, A.J.; Bijker, E.M. A guide to vaccinology: From basic principles to new developments. Nat. Rev. Immunol. 2021, 21, 83-100. [CrossRef]

48. Riva, L.; Yuan, S.; Yin, X.; Martin-Sancho, L.; Matsunaga, N.; Pache, L.; Muehlbacher, B.S.; Jesus, D.P.D.; Teriete, P.; Hull, M.V.; et al. Discovery of SARS-CoV-2 anti-viral drugs through large-scale compound repurposing. Nature 2020, 586, 113-119. [CrossRef]

49. Bhuiyan, F.R.; Howlader, S.; Raihan, T.; Hasan, M. Plants Metabolites: Possibility of Natural Therapeutics against the COVID-19 Pandemic. Front. Med. (Lausanne) 2020, 7, 444. [CrossRef]

50. Mahmood, N.; Nasir, S.B.; Hefferon, K. Plant-Based Drugs and Vaccines for COVID-19. Vaccines (Basel) 2020, 9, 15. [CrossRef]

51. Khanna, K.; Kohli, S.K.; Kaur, R.; Bhardwaj, A.; Bhardwaj, V.; Ohri, P.; Sharma, A.; Ahmad, A.; Bhardwaj, R.; Ahmad, P. Herbal immune-boosters: Substantial warriors of pandemic COVID-19 battle. Phytomedicine 2021, 85, 153361. [CrossRef]

52. Omrani, M.; Keshavarz, M.; Ebrahimi, N.S.; Mehrabi, M.; McGaw, L.J.; Abdalla, A.M.; Mehrbod, P. Potential Natural Products Against Respiratory Viruses: A Perspective to Develop Anti-COVID-19 Medicines. Front. Pharmacol. 2021, 11, 586993. [CrossRef]

53. Mei, M.; Tan, X. Current Strategies of Antiviral Drug Discovery for COVID-19. Front. Mol. Biosci. 2021, 8, 671263. [CrossRef]

54. Rehman, M.F.U.; Akhter, S.; Batool, A.I.; Selamoglu, Z.; Sevindik, M.; Eman, R.; Mustaqeem, M.; Akram, M.S.; Kanwal, F.; Lu, C.; et al. Effectiveness of Natural Antioxidants against SARS-CoV-2? Insights from the In-Silico World. Antibiotics 2021, 10, 1011. [CrossRef]

55. Joshi, C.; Chaudhari, A.; Joshi, C.; Joshi, M.; Bagatharia, S. Repurposing of the herbal formulations: Molecular docking and molecular dynamics simulation studies to validate the efficacy of phytocompounds against SARS-CoV-2 proteins. J. Biomol. Struct. Dyn. 2021, 14, 1-15. [CrossRef] [PubMed]

56. Ali, G.S.; Ozdemir, B.; Selamoglu, Z. A Review of Severe Acute Respiratory Syndrome Coronavirus 2 and Pathological Disorders in Patients. J. Pharm. Care 2021, 9, 141-147. [CrossRef]

57. Thas, J.J. Siddha medicine-Background and principles and the application for skin diseases. Clin. Dermatol. 2008, 26, 62-78. [CrossRef]

58. Kumar, D.; Arya, V.; Kaur, R.; Bhat, Z.A.; Gupta, V.K.; Kumar, V. A review of immunomodulators in the Indian traditional health care system. J. Microbiol. Immunol. Infect. 2012, 45, 165-184. [CrossRef] [PubMed]

59. Karunamoorthi, K.; Jegajeevanram, K.; Xavier, J.; Vijayalakshmi, J.; Melita, L. Tamil traditional medicinal system-Siddha: An indigenous health practice in the international perspectives. Tang 2012, 2,1-11. [CrossRef] 
60. Natarajan, S.; Anbarasi, C.; Sathiyarajeswaran, P.; Manickam, P.; Geetha, S.; Kathiravan, R.; Prathiba, P.; Pitchiahkumar, M.; Parthiban, P.; Kanakavalli, K.; et al. Kabasura Kudineer (KSK), a poly-herbal Siddha medicine, reduced SARS-CoV-2 viral load in asymptomatic COVID-19 individuals as compared to vitamin C and zinc supplementation: Findings from a prospective, exploratory, open-labeled, comparative, randomized controlled trial, Tamil Nadu, India. Trials 2021, 22, 623. [PubMed]

61. Natarajan, S.; Anbarasi, C.; Sathiyarajeswaran, P.; Manickam, P.; Geetha, S.; Kathiravan, R.; Prathiba, P.; Pitchiahkumar, M.; Parthiban, P.; Kanakavalli, K.; et al. The efficacy of Siddha Medicine, Kabasura Kudineer (KSK) compared to Vitamin C \& Zinc (CZ) supplementation in the management of asymptomatic COVID-19 cases: A structured summary of a study protocol for a randomised controlled trial. Trials 2020, 21, 892. [PubMed]

62. Srivastava, A.; Rengaraju, M.; Srivastava, S.; Narayanan, V.; Gupta, V.; Upadhayay, R.; Kumar, J.; Parameswaran, S.; Kadarkarai, K.; Velmurugan, A. Efficacy of two siddha polyherbal decoctions, Nilavembu Kudineer and Kaba Sura Kudineer, along with standard allopathy treatment in the management of mild to moderate symptomatic COVID-19 patients-a double-blind, placebo-controlled, clinical trial. Trials 2021, 22, 570. [CrossRef] [PubMed]

63. Vincent, S.; Arokiyaraj, S.; Saravanan, M.; Dhanraj, M. Molecular Docking Studies on the Anti-viral Effects of Compounds from Kabasura Kudineer on SARS-CoV-2 3CLpro. Front. Mol. Biosci. 2020, 7, 613401. [CrossRef] [PubMed]

64. Nallusamy, S.; Mannu, J.; Ravikumar, C.; Angamuthu, K.; Nathan, B.; Nachimuthu, K.; Ramasamy, G.; Muthurajan, R.; Subbarayalu, M.; Neelakandan, K. Exploring Phytochemicals of Traditional Medicinal Plants Exhibiting Inhibitory Activity Against Main Protease, Spike Glycoprotein, RNA-dependent RNA Polymerase and Non-Structural Proteins of SARS-CoV-2 Through Virtual Screening. Front. Pharmacol. 2021, 12, 667704. [CrossRef]

65. Lau, K.M.; Lee, K.M.; Koon, C.M.; Cheung, C.S.; Lau, C.P.; Ho, H.M.; Lee, M.Y.; Au, S.W.; Cheng, C.H.; Lau, C.B.; et al. Immunomodulatory and anti-SARS activities of Houttuynia cordata. J. Ethnopharmacol. 2008, 118, 79-85. [CrossRef]

66. Shanmugaraj, B.; Siriwattananon, K.; Wangkanont, K.; Phoolcharoen, W. Perspectives on monoclonal antibody therapy as potential therapeutic intervention for Coronavirus disease-19 (COVID-19). Asian Pac. J. Allergy Immunol. 2020, 38, 10-18.

67. Wu, Z.; Deng, X.; Hu, Q.; Xiao, X.; Jiang, J.; Ma, X.; Wu, M. Houttuynia cordata Thunb: An Ethnopharmacological Review. Front. Pharmacol. 2021, 12, 714694. [CrossRef]

68. Das, S.K.; Mahanta, S.; Tanti, B.; Tag, H.; Hui, P.K. Identification of phytocompounds from Houttuynia cordata Thunb. as potential inhibitors for SARS-CoV-2 replication proteins through GC-MS/LC-MS characterization, molecular docking and molecular dynamics simulation. Mol. Divers. 2021, 7, 1-24. [CrossRef]

69. Bahramsoltani, R.; Rahimi, R. An Evaluation of Traditional Persian Medicine for the Management of SARS-CoV-2. Front. Pharmacol. 2020, 11, 571434. [CrossRef] [PubMed]

70. Vicidomini, C.; Roviello, V.; Roviello, G.N. Molecular Basis of the Therapeutical Potential of Clove (Syzygium aromaticum L.) and Clues to Its Anti-COVID-19 Utility. Molecules 2021, 26, 1880. [CrossRef] [PubMed]

71. Tallei, T.E.; Tumilaar, S.G.; Niode, N.J.; Kepel, F.B.J.; Idroes, R.; Effendi, Y.; Sakib, S.A.; Emran, T.B. Potential of Plant Bioactive Compounds as SARS-CoV-2 Main Protease (Mpro) and Spike (S) Glycoprotein Inhibitors: A Molecular Docking Study. Scientifica 2020, 2020, 6307457. [CrossRef]

72. Khazdair, M.R.; Ghafari, S.; Sadeghi, M. Possible therapeutic effects of Nigella sativa and its thymoquinone on COVID-19. Pharm. Biol. 2021, 59, 696-703. [CrossRef]

73. Kulyar, M.F.; Li, R.; Mehmood, K.; Waqas, M.; Li, K.; Li, J. Potential influence of Nagella sativa (Black cumin) in reinforcing immune system: A hope to decelerate the COVID-19 pandemic. Phytomedicine 2021, 85, 153277. [CrossRef] [PubMed]

74. Ghoran, S.H.; El-Shazly, M.; Sekeroglu, N.; Kijjoa, A. Natural Products from Medicinal Plants with Anti-Human Coronavirus Activities. Molecules 2021, 26, 1754. [CrossRef]

75. Shree, P.; Mishra, P.; Selvaraj, C.; Singh, S.K.; Chaube, R.; Garg, N.; Tripathi, Y.B. Targeting COVID-19 (SARS-CoV-2) main protease through active phytochemicals of ayurvedic medicinal plants_Withania somnifera (Ashwagandha), Tinospora cordifolia (Giloy) and Ocimum sanctum (Tulsi)-A molecular docking study. J. Biomol. Struct. Dyn. 2020, 27, 1-14. [CrossRef] [PubMed]

76. Nag, A.; Chowdhury, R.R. Piperine, an alkaloid of black pepper seeds can effectively inhibit the anti-viral enzymes of Dengue and Ebola viruses, an in silico molecular docking study. Virusdisease 2020, 31, 308-315. [CrossRef] [PubMed]

77. Davella, R.; Gurrapu, S.; Mamidala, E. Phenolic compounds as promising drug candidates against COVID-19-An integrated molecular docking and dynamics simulation study. Mater. Today Proc. 2021. [CrossRef]

78. Khanal, P.; Duyu, T.; Patil, B.M.; Dey, Y.N.; Pasha, I.; Wanjari, M.; Gurav, S.S.; Maity, A. Network pharmacology of AYUSH recommended immune-boosting medicinal plants against COVID-19. J. Ayurveda Integr. Med. 2020, 13, 100374. [CrossRef] [PubMed]

79. Metastasio, A.; Prevete, E.; Singh, D.; Grundmann, O.; Prozialeck, W.C.; Veltri, C.; Bersani, G.; Corazza, O. Can Kratom (Mitragyna speciosa) Alleviate COVID-19 Pain? A Case Study. Front. Psychiatry 2020, 11, 594816. [CrossRef]

80. Dallakyan, S.; Olson, A.J. Small-molecule library screening by docking with PyRx. Methods Mol. Biol. 2015, 1263, 243-250. [PubMed]

81. Baugh, E.H.; Lyskov, S.; Weitzner, B.D.; Gray, J.J. Real-time PyMOL visualization for Rosetta and PyRosetta. PLoS ONE 2011, 6, e21931. [CrossRef] [PubMed]

82. Laskowski, R.A.; Swindells, M.B. LigPlot+: Multiple ligand-protein interaction diagrams for drug discovery. J. Chem. Inf. Model. 2011, 51, 2778-2786. [CrossRef] [PubMed]

83. Xiong, G.; Wu, Z.; Yi, J.; Fu, L.; Yang, Z.; Hsieh, C.; Yin, M.; Zeng, X.; Wu, C.; Lu, A.; et al. ADMETlab 2.0: An integrated online platform for accurate and comprehensive predictions of ADMET properties. Nucleic Acids Res. 2021, 49, W5-W14. [CrossRef] 
84. Banerjee, P.; Eckert, A.O.; Schrey, A.K.; Preissner, R. ProTox-II: A webserver for the prediction of toxicity of chemicals. Nucleic Acids Res. 2018, 46, W257-W263. [CrossRef] [PubMed]

85. Saravanan, J.; Devasia, N.; Gopalasatheeshkumar, K.; Devan, S.V.; Koikila, T.K.; Sanjay, M. Anti-inflammatory, antipyretic and antibacterial study of Kabasura kudineer choornam. Int. J. Curr. Adv. Res. 2018, 7, 9992-9997.

86. Meenakumari, R.; Thangaraj, K.; Sundaram, A.; Sundaram, M.M.; Shanmugapriya, P.; Mariappan, A.; George, M.; Suba, V.; Rajalakshmi, E.; Sendhilkumar, M. Clinical outcomes among COVID-19 patients managed with modern and traditional Siddha medicine -A retrospective cohort study, Chennai, Tamil Nadu, India, 2020. J. Ayurveda Integr. Med. 2021. [CrossRef] [PubMed]

87. Ma, J.; Su, D.; Sun, Y.; Huang, X.; Liang, Y.; Fang, L.; Ma, Y.; Li, W.; Liang, P.; Zheng, S. Cryo-EM structure of S-Trimer, a subunit vaccine candidate for COVID-19. J. Virol. 2021, 95, e00194-e21. [CrossRef]

88. O'Boyle, N.M.; Banck, M.; James, C.A.; Morley, C.; Vandermeersch, T.; Hutchison, G.R. Open Babel: An open chemical toolbox. J. Cheminform. 2011, 3, 33. [CrossRef]

89. Trott, O.; Olson, A.J. AutoDock Vina: Improving the speed and accuracy of docking witha new scoring function, efficient optimization, and multithreading. J. Comput. Chem. 2010, 31, 455-461. [PubMed]

90. Hirschhorn, H.H. Botanical remedies of the former Dutch East Indies (Indonesia): Part I: Eumycetes, pteridophyta, gymnospermae, angiospermae (monocotyledones only). J. Ethnopharm. 1983, 7, 123-156. [CrossRef]

91. Ren, X.; Sui, X.; Yin, J. The effect of Houttuynia cordata injection on pseudorabies herpes virus (PrV) infection in vitro. Pharma. Biol. 2011, 49, 161-166. [CrossRef]

92. Leardkamolkarn, V.; Sirigulpanit, W.; Phurimsak, C.; Kumkate, S.; Himakoun, L.; Sripanidkulchai, B. The inhibitory actions of Houttuynia cordata aqueous extract on dengue virus and dengue infected cells. J. Food Biochem. 2012, 36, 86-92. [CrossRef]

93. Jiangang, F.; Ling, D.; Zhang, L.; Hongmei, L. Houttuynia cordata Thunb: A review of phytochemistry and pharmacology and quality control. Chin. Med. 2013, 4, 101-123.

94. Hung, P.Y.; Ho, B.C.; Lee, S.Y.; Chang, S.Y.; Kao, C.L.; Lee, S.S.; Lee, C.N. Houttuynia cordata targets the beginning stage of herpes simplex virus infection. PLoS ONE 2015, 10, e0115475. [CrossRef]

95. Satthakarn, S.; Chung, W.O.; Promsong, A.; Nittayananta, W. Houttuynia cordata modulates oral innate immune mediators: Potential role of herbal plant on oral health. Oral Dis. 2015, 21, 512-518. [CrossRef]

96. Sit, N.W.; Chan, Y.S.; Chuah, B.L.; Cheng, R.J.; Leong, W.M.; Khoo, K.S. Anti-viral, antifungal and antibacterial activities of the Chinese medicinal plants, Houttuynia cordata, Lobelia chinensis and Selaginella uncinata. Southeast Asian J. Trop. Med. Public Health 2018, 48, 616-627.

97. Cheng, D.; Sun, L.; Zou, S.; Chen, J.; Mao, H.; Zhang, Y. Anti-viral effects of Houttuynia cordata polysaccharide extract on Murine Norovirus-1 (MNV-1): A human norovirus surrogate. Molecules 2019, 24, 1835. [CrossRef]

98. Ling, L.J.; Lu, Y.; Zhang, Y.Y.; Zhu, H.Y.; Tu, P.; Li, H.; Chen, D.F. Flavonoids from Houttuynia cordata attenuate H1N1-induced acute lung injury in mice via inhibition of influenza virus and Toll-like receptor signalling. Phytomedicine 2020, 67, 153150. [CrossRef]

99. Fawe, A.; Zaid, M.A.; Menzies, J.G.; Bélanger, R.R. Silicon-Mediated Accumulation of flavonoid Phytoalexins in Cucumber. J. Phytopathol. 1998, 88, 396-401. [CrossRef]

100. Banerjee, S.; Panda, C.K.; Das, S. Clove (Syzygium aromaticum L.), a potential chemopreventive agent for lung cancer. Carcinogenesis 2006, 27, 1645-1654. [CrossRef]

101. Horiuchi, K.; Shiota, S.; Hatano, T.; Yoshida, T.; Kuroda, T.; Tsuchiva, T. Antimicrobial activity of oleanolic acid from Salvia officinalis and related compounds on vancomycin-resistant enterococci (VRE). J. Biol. Pharm. Bull. 2007, 30, 1147-1149. [CrossRef]

102. Feng, J.H.; Chen, W.; Zhao, Y.; Ju, X.L. Antitumor activity of Oleanolic, Ursolic and Glycyrrhetinic acid. Open Nat. Prod. J. 2009, 2, 48-52. [CrossRef]

103. Bhuiyan, M.N.I.; Begum, J.; Nandi, N.C.; Akter, F. Constituents of the essential oil from leaves and buds of clove (Syzygium caryophyllatum (L.) Alston). Afr. J. Plant. Sci. 2010, 4, 451-454.

104. Vasconellos, M.C.; Benzerra, D.P.; Fonseca, A.M.; Pessoa, C.; Lemos, T.L.; Lotufo, C.L.V.; de Moraes, M.O.; Montenegro, R.C. The in vitro and in vivo inhibitory activity of biflorin in melanoma. J. Melanoma Res. 2011, 21, 106-114. [CrossRef]

105. Sánchez-Tena, S.; Reyes-Zurita, F.J.; Díaz-Moralli, S.; Vinardell, M.P.; Reed, M.; García-García, F.; Dopazo, J.; Lupiáñez, J.A.; Günther, U.; Cascante, M. Maslinic acid-enriched diet decreases intestinal tumorigenesis in Apc (Min/+) mice through transcriptomic and metabolomic reprogramming. PLoS ONE 2013, 8, e59392.

106. Castellano, J.M.; Guinda, A.; Deloado, T.; Rada, M.; Cavuela, J.A. Biochemical basis of the antidiabetic activity of oleanolic acid and related pentacyclic triterpenes. J. Diabetes 2013, 62, 1791-1799. [CrossRef] [PubMed]

107. Liu, H.; Schmitz, J.C.; Wei, J.; Cao, S.; Beumer, J.H.; Strychor, S.; Cheng, L.; Liu, M.; Wang, C.; Wu, N.; et al. Clove extract inhibits tumor growth and promotes cell cycle arrest and apoptosis. Oncol. Res. 2014, 21, 247-259. [CrossRef]

108. Jnawali, H.N.; Lee, E.; Jeong, K.W.; Shin, A.; Heo, Y.S.; Kim, Y. Anti-inflammatory Activity of Rhamnetin and a Model of Its Binding to c-Jun NH-Terminal Kinase 1 and p38 MAPK. J. Nat. Prod. 2014, 77, 258-263. [CrossRef]

109. Park, E.S.; Kang, J.C.; Jang, Y.C.; Park, J.S.; Jang, S.Y.; Kim, D.E.; Kim, B.; Shin, H.S. Cardioprotective effects of rhamnetin in H9c2 cardiomyoblast cells under $\mathrm{H}_{2} \mathrm{O}_{2}$-induced apoptosis. J. Ethnopharmacol. 2014, 153, 552-560. [CrossRef]

110. Mossadeq, S.W.M.; Sulaiman, M.R.; Mohamad, T.T.A.; Chiong, H.S.; Zakaria, Z.A.; Jabit, M.L.; Israf, D.A. Anti-inflammatory and antinociceptive effects of Mitragyna speciosa Korth methanolic extract. Med. Princ. Pract. 2009, 18, 378-384. [CrossRef] [PubMed] 
111. Parthasarathy, S.; Azizi, J.; Ramanathan, B.S.; Ismail, S.; Sasidharan, S.; Mohd, M.I.; Mansor, S.M. Evaluation of antioxidant and antibacterial activities of aqueous, methanolic and alkaloid extracts from Mitragyna speciosa (rubiaceae family) leaves. Molecules 2009, 14, 3964-3974. [CrossRef] [PubMed]

112. Azizi, J.; Ismail, S.; Mordi, M.N.; Ramanathan, S.; Said, M.I.M.; Mansor, S.M. In vitro and in vivo effects of three different mitragyna speciosa korth leaf extracts on phase II drug metabolizing enzymes-glutathione transferases (GSTs). Molecules 2010, 15, 432-441. [CrossRef] [PubMed]

113. Goh, T.B.; Yian, K.R.; Mordi, M.N.; Mansor, S.M. Antioxidant value and antiproliferative efficacy of mitragynine and a silane reduced analougue. Asian Pac. J. Cancer Prev. 2014, 15, 5659-5665. [CrossRef]

114. Srichana, K.; Janchawee, B.; Prutipanlai, S.; Raungrut, P.; Keawpradub, N. Effects of mitragynine and a crude alkaloid extract derived from Mitragyna speciosa korth. On permethrin elimination in rats. Pharmaceutics 2015, 7, 10-26. [CrossRef]

115. Goh, Y.S.; Karunakaran, T.; Murugaiyah, V.; Santhanam, R.; Abu Bakar, M.H.; Ramanathan, S. Accelerated Solvent Extractions (ASE) of Mitragyna speciosa Korth. (Kratom) Leaves: Evaluation of Its Cytotoxicity and Antinociceptive Activity. Molecules 2021, 26, 3704. [CrossRef]

116. Singh, M.K.; Khare, G.; Iyer, S.K.; Sharwan, G.; Tripathi, D.K. Clerodendrum serratum: A clinical approach. J. App. Pharm. Sci. 2012, 2,11-15.

117. Patel, J.J.; Acharya, S.R.; Acharya, N.S. Clerodendrum serratum (L.) Moon.-A review on traditional uses, phytochemistry and pharmacological activities. Ethnopharmacology 2014, 154, 268-285. [CrossRef]

118. Kshirsagar, A.D.; Ingale, K.G.; Vyawahare, N.S.; Thorve, V.S. Hygrophila spinosa: A comprehensive review. Pharmacogn. Rev. 2010, 4, 167-171. [CrossRef]

119. Salve, S.D.; Bhuktar, A.S. Pharmacognosy and phytochemical evaluation of Hygrophila auriculata (Schumach.) heine root. J. Phytopharm. 2017, 6, 210-216. [CrossRef]

120. Sodhi, T. Ayurveda in Veterinary Medicine. In Proceedings of the AHVMA Annual Conference, Durham, NC, USA, 20-23 September 2003.

121. Kirtikar, K.R.; Basu, B.D. Indian Medicinal Plants, 1st ed.; Bio-green Books: Delhi, India, 1987; pp. 757-759.

122. Shobana, S.; Naidu, A.K. Antioxidant activity of selected Indian spices. Prostaglandins Leukot. Essent. Fat. Acids. 2000, 62, 107-110. [CrossRef] [PubMed]

123. Waisundara, V.Y.; Watawana, M.I.; Jayawardena, N. Costus speciosus and Coccinia grandis: Traditional medicinal remedies for diabetes. S. Afr. J. Bot. 2015, 98, 1-5. [CrossRef]

124. Selim, S.; Jaouni, S.A. Anticancer and apoptotic effects on cell proliferation of diosgenin isolated from Costus speciosus (Koen.) Sm. BMC Complementary Altern. Med. 2015, 15, 301. [CrossRef] [PubMed]

125. El-Far, A.H.; Shaheen, H.M.; Alsenosy, A.W.; El-Sayed, Y.S.; Al Jaouni, S.K.; Mousa, S.A. Costus speciosus: Traditional uses, phytochemistry, and therapeutic potentials. Pharmacogn. Rev. 2018, 12, 120-127. [CrossRef]

126. Ma, S.C.; Du, J.; But, P.P.; Deng, X.L.; Zhang, Y.W.; Ooi, V.E.; Xu, H.X.; Lee, S.H.; Lee, S.F. Antiviral Chinese medicinal herbs against respiratory syncytial virus. J. Ethnopharmacol. 2002, 79, 205-211. [CrossRef]

127. Jadhav, A.K.; Karuppayil, S.M. Andrographis paniculata (Burm. F) Wall ex Nees: Anti-viral properties. Phytother. Res. 2021, 127, 5365-5373. [CrossRef]

128. Aglin, A.A. Medicinal effects of Mollugo cerviana-A Review. Int. J. Sci. Res. Multidiscip. Stud. 2018, 4, 34-37.

129. Napagoda, M.; Gerstmeier, J.; Butschek, H.; De Soyza, S.; Pace, S.; Lorenz, S.; Qader, M.; Witharana, S.; Nagahawatte, A.; Wijayaratne, G.; et al. The Anti-Inflammatory and Antimicrobial Potential of Selected Ethnomedicinal Plants from Sri Lanka. Molecules 2020, 25, 1894. [CrossRef]

130. Reddy, B.S.; Rao, N.R.; Vijeepallam, K.; Pandy, V. Phytochemical, pharmacological and biological profiles of Tragia species (Family: Euphorbiaceae). Afr. J. Tradit. Complement Altern. Med. 2017, 14, 105-112. [CrossRef]

131. Zhao, X.; Chen, H.; Wang, H. Glycans of SARS-CoV-2 Spike Protein in Virus Infection and Antibody Production. Front. Mol. Biosci. 2021, 8, 629873. [CrossRef] [PubMed]

132. Baral, P.; Bhattarai, N.; Hossen, M.L.; Stebliankin, V.; Gerstman, B.S.; Narasimhan, G.; Chapagain, P.P. Mutation-induced changes in the receptor-binding interface of the SARS-CoV-2 Delta variant B.1.617.2 and implications for immune evasion. Biochem. Biophys. Res. Commun. 2021, 574, 14-19. [CrossRef] [PubMed]

133. Motozono, C.; Toyoda, M.; Zahradnik, J.; Saito, A.; Nasser, H.; Tan, T.S.; Ngare, I.; Kimura, I.; Uriu, K.; Kosugi, Y.; et al. SARS-CoV-2 spike L452R variant evades cellular immunity and increases infectivity. Cell Host Microbe 2021, 29, 1124-1136.e11. [CrossRef] [PubMed]

134. Tchesnokova, V.; Kulakesara, H.; Larson, L.; Bowers, V.; Rechkina, E.; Kisiela, D.; Sledneva, Y.; Choudhury, D.; Maslova, I.; Deng, K.; et al. Acquisition of the L452R mutation in the ACE2-binding interface of Spike protein triggers recent massive expansion of SARS-CoV-2 variants. J. Clin. Microbiol. 2021, 11, JCM0092121. [CrossRef]

135. Mouffouk, C.; Mouffouk, S.; Mouffouk, S.; Hambaba, L.; Haba, H. Flavonols as potential anti-viral drugs targeting SARS-CoV-2 proteases (3CLpro and PLpro), spike protein, RNA-dependent RNA polymerase (RdRp) and angiotensin-converting enzyme II receptor (ACE2). Eur. J. Pharmacol. 2021, 891, 173759. [CrossRef]

136. Rosales-Mendoza, S.; Márquez-Escobar, V.A.; González-Ortega, O.; Nieto-Gómez, R.; Arévalo-Villalobos, J.I. What Does PlantBased Vaccine Technology Offer to the Fight against COVID-19? Vaccines 2020, 8, 183. [CrossRef]

137. LeBlanc, Z.; Waterhouse, P.; Bally, J. Plant-Based Vaccines: The Way Ahead? Viruses 2020, 13, 5. [CrossRef] [PubMed] 
138. Das, A.; Pandita, D.; Jain, G.K.; Agarwal, P.; Grewal, A.S.; Khar, R.K.; Lather, V. Role of phytoconstituents in the management of COVID-19. Chem. Biol. Interact. 2021, 341, 109449. [CrossRef] [PubMed]

139. Chojnacka, K.; Witek-Krowiak, A.; Skrzypczak, D.; Mikula, K.; Młynarz, P. Phytochemicals containing biologically active polyphenols as an effective agent against COVID-19-inducing coronavirus. J. Funct. Foods. 2020, 73, 104146. [CrossRef]

140. Anand, A.V.; Balamuralikrishnan, B.; Kaviya, M.; Bharathi, K.; Parithathvi, A.; Arun, M.; Senthilkumar, N.; Velayuthaprabhu, S.; Saradhadevi, M.; Al-Dhabi, N.A.; et al. Medicinal Plants, Phytochemicals, and Herbs to Combat Viral Pathogens Including SARS-CoV-2. Molecules 2021, 26, 1775. [CrossRef]

141. RECOVERY Collaborative Group. Lopinavir-ritonavir in patients admitted to hospital with COVID-19 (RECOVERY): A randomised, controlled, open-label, platform trial. Lancet 2020, 396, 1345-1352. [CrossRef]

142. Jorgensen, S.C.J.; Kebriaei, R.; Dresser, L.D. Remdesivir: Review of Pharmacology, Pre-clinical Data, and Emerging Clinical Experience for COVID-19. Pharmacotherapy 2020, 40, 659-671. [CrossRef]

143. Touret, F.; de Lamballerie, X. Of chloroquine and COVID-19. Antivir. Res. 2020, 177, 104762. [CrossRef]

144. Nojomi, M.; Yassin, Z.; Keyvani, H.; Makiani, M.J.; Roham, M.; Laali, A.; Dehghan, N.; Navaei, M.; Ranjbar, M. Effect of Arbidol (Umifenovir) on COVID-19: A randomized controlled trial. BMC Infect. Dis. 2020, 20, 954. [CrossRef]

145. Joshi, S.; Parkar, J.; Ansari, A.; Vora, A.; Talwar, D.; Tiwaskar, M.; Patil, S.; Barkate, H. Role of favipiravir in the treatment of COVID-19. Int. J. Infect. Dis. 2021, 102, 501-508. [CrossRef]

146. Hung, I.F.; Lung, K.C.; Tso, E.Y.; Liu, R.; Chung, T.W.; Chu, M.Y.; Ng, Y.Y.; Lo, J.; Chan, J.; Tam, A.R.; et al. Triple combination of interferon beta-1b, lopinavir-ritonavir, and ribavirin in the treatment of patients admitted to hospital with COVID-19: An open-label, randomised, phase 2 trial. Lancet 2020, 395, 1695-1704. [CrossRef]

147. Boulware, D.R.; Pullen, M.F.; Bangdiwala, A.S.; Pastick, K.A.; Lofgren, S.M.; Okafor, E.C.; Skipper, C.P.; Nascene, A.A.; Nicol, M.R.; Abassi, M.; et al. A Randomized Trial of Hydroxychloroquine as Postexposure Prophylaxis for COVID-19. N. Engl J. Med. 2020, 383, 517-525. [CrossRef]

148. Nourian, A.; Khalili, H. Sofosbuvir as a potential option for the treatment of COVID-19. Acta Biomed. 2020, 91, 236-238.

149. Kallingal, A.; Thachan Kundil, V.; Ayyolath, A.; Karlapudi, A.P.; Muringayil Joseph, T.; E, J.V. Molecular modeling study of tectoquinone and acteoside from Tectona grandis linn: A new SARS-CoV-2 main protease inhibitor against COVID-19. J. Biomol. Struct. Dyn. 2020, 9, 1-12. [CrossRef]

150. Shawky, E.; Nada, A.A.; Ibrahim, R.S. Potential role of medicinal plants and their constituents in the mitigation of SARS-CoV-2 Identifying related therapeutic targets using network pharmacology and molecular docking analyses. RSC Adv. 2020, 10, 27961-27983. [CrossRef]

151. Mbaveng, A.T.; Ngameni, B.; Kuete, V.; Simo, I.K.; Ambassa, P.; Roy, R.; Bezabih, M.; Etoa, F.X.; Ngadjui, B.T.; Abegaz, B.M.; et al. Antimicrobial activity of the crude extracts and five flavonoids from the twigs of Dorstenia barteri (Moraceae). J. Ethnopharmacol. 2008, 116, 483-489. [CrossRef] [PubMed]

152. Benet, L.Z.; Hosey, C.M.; Ursu, O.; Oprea, T.I. BDDCS, the Rule of 5 and drugability. Adv. Drug Deliv. Rev. 2016, 101, 89-98. [CrossRef] [PubMed]

153. Guidelines for Ayurveda Practitioners for COVID 19. Available online: https://vikaspedia.in/health/ayush/guidelines-forayush-practitioners-for-covid-19/guidelines-for-ayurveda-practitioners-for-covid19 (accessed on 11 November 2021).

154. World Health Organization. 2020. Available online: https://www.who.int/publications/m/item/weekly-epidemiologicalupdate---19-january-2021 (accessed on 11 November 2021).

155. Gautam, S.; Gautam, A.; Chhetri, S.; Bhattarai, U. Immunity against COVID-19: Potential Role of Ayush Kwath. J. Ayurveda Integr. Med. 2020, 100350. [CrossRef] [PubMed] 